BEHAVIORAL FOUNDATIONS OF RECIPROCITY:

-EXPRIMENTAI ECONOMICS AND EVOLUIIONARY PSYCHOLOG

Elizabeth Hoffman, Iowa State University

Kevin McCabe, University of Minnesota vernon Smith, University of Arizona

August, 1995 


\title{
BEHAVIORAL FOUNDATIONS OF RECIPROCITY : EXPERIMENTAL ECONOMICS AND EVOLUTIONARY PSYCHOLOGY
}

\author{
Elizabeth Hoffman, Iowa State University \\ Kevin McCabe, University of Minnesota \\ Vernon Smith, University of Arizona
}

\begin{abstract}
Laboratory experiments have generally supported the fundamental theorem that, in classical property rights environments, noncooperative behavior in large group markets yields efficient social outcomes. Experiments, however, regularly fail to support the game theoretic prediction of noncooperative behavior in small group strategic interaction and in public good environments. In these two types of experiments subjects frequently achieve more efficient social outcomes they collect more' money from the experimenter - than noncooperative game theory predicts. As we interpret it, subject behavior in these experiments exhibits a habit of reciprocity even in single-play games. Evolutionary psychologists hypothesize that this is because humans have evolved mental algorithms for identifying and punishing cheaters who behave non cooperatively in social exchange.

For about 2-3 million years humans have lived in small interactive groups, and this, has required adaptation to the fitness demands of social exchange. The hypothesis follows that the human mind is composed of context-specific mental modules that operate on the cost-benefit characteristics of social exchange. This requires the mind to be adept at detecting cheating on implied or explicit social exchange contracts. hypothesis is contrary to game and economic theories which formally develop a small number of domain general principles of strategic interaction, which are applicable across all strategically similar contexts.

Evolutionary psychologists have reported an impressive number of individual. decision making experiments designed to test competing hypotheses about human cognition rules in social exchange. We build on this work, and extend $i t$ as an organizing principle to examine and explain subject behavior in public good, ultimatum, dictator, and more general extensive form bargaining games.
\end{abstract}


I. Introduction

Economists have long studied the fundamental problem that cooperative outcomes, that make agents as a whole better of than a 11 other outcomes, generally cannot be supported as equilibria in finite games (see Fudenberg and Tirole, 1993; Osborne and Rubinstein, 1994; Ostrom, Gardner, and Walker, 1994). This is often referred to as the Prisoners Dilemma, the social dilemma, or the free rider problem. Recognition of this problem has led to an extensive theoretical and experimental literature on incentive-compatible mechanisms for the allocation of public and externality goods. In addition, economists focus policy recommendations on the development of incentives for individuals to behave cooperatively, assuming it is not in their nature to do so. (See, e.g., Dasgupta, Hammond, and Maskin, 1979; Fudenberg and Tircle, 1993; Green and Laffont, 1979; Groves, 1973; Laffont, 1994) .

While there are numerous examples of noncooperative behavior in the economy and the laboratory, the puzzle for economists is the prevalence of small group cooperative behavior in the absence of any obvious incentives to engage in such behavior. Thus, in large group experimental markets among anonymous agents in private property regimes noncooperative behavior yields efficient outcomes. But in two-person bargaining experiments under the same anonymity conditions, where noncooperative behavior does not support efficient outcomes, we observe more cooperative behavior than such environments are expected to produce. Moreover, 
examples of the achievement of cooperative behavior by decentralized means have a long history in the human experience. Anthropological and archeological evidence suggest that sharing behavior is ubiquitous in primitive cultures that lack markets, monetary systems, or other means of storing and redistributing wealth (see, e.g., Cosmides and Tooby, 1987, 1989; Isaac, 1978; Kaplin and Hill, 1985; Lee and De Vore, 1968; Tooby and De Vore, 1987; Trivers, 1971). In medieval Europe, peasants, engaged in common-field agriculture, established "stint-rights" in pasture to control potential overgrazing, and elaborate methods, based upon labor and capital inputs by each household, for determining the shares of common output claimed by each household (Macfarlane, 1978). Through extensive field research, Ostrom and her colleagues at Indiana University have identified many contemporary and ancient examples of decentralized cooperative solutions to public good and externality problems (Ostrom, Gardner, and Walker, 1994). During the midwestern flood of 1993 communities across the midwest came together to build and shore up levies, sandbag homes and businesses, and clean up the mess after the flood waters receded.

In this paper we draw together theoretical and experimental evidence from repeated game theory, evolutionary psychology, and experimental economics that provides the basis for developing a framework for understanding the persistence of cooperative outcomes in the face of contrary individual incentives. Repeated game theory with discounting or infinite time horizons allows for 
cooperative solutions, but does not allow researchers to predict that cooperative solutions will prevail (Fudenberg and Tirole, 1993). Recent research in evolutionary psychology (Cosmides and Tooby, 1987, 1989, 1992) suggests that humans may be evolutionarily predisposed to engage in social exchange using mental algorithms that identify and punish cheaters. Finally, a considerable body of research in experimental economics now identifies a number of environmental and institutional factors that promote cooperation even, in the face of contrary individual incentives (Davis and Holt, 1993; Isac and Walker, 1988a,b, 1991; Isaac, Walker, and Thomas, 1984; Isaac, Walker, and Williams, 1991).

\section{I . Repeated Games}

The Prisoners' Dilemma game shown in Figure 1 demonstrates the problem of achieving cooperation. Row and Column each have a dominant strategy to choose $D$ (defect) even though the resulting payoff $(0,0)$ is Pareto dominated by $(3,3)$.

\begin{tabular}{|l|c|c|c|}
\hline \multirow{2}{*}{$\begin{array}{c}\text { Prisoner's Dilemma } \\
\text { Game }\end{array}$} & \multicolumn{2}{|c|}{ Column } & Player \\
\hline Row & C & D \\
Player & C & $(3,3)$ & $(-5,5)$ \\
\hline
\end{tabular}

Figure 1 
However, when players interact more than once they can consider strategies in which current decisions depend on past play. For example, the strategy tit-for-tat (Axelrod 1984) cooperates in the first period, and then copies its counterpart's move from the previous period. The study of repeated games has provided game theorists with the following explanations of cooperation based on self-interest: self-enforcing equilibria, and reputations. Each of these explanations is discussed below.

Self-enforcing equilibria are based on the idea that players can credibly punish non-cooperative defections. Suppose, for example, that $i t$ is common knowledge that the PrisonersDilemma, shown in Figure 1 , is to be followed by the coordination game shown in Figure 2 .

\begin{tabular}{|c|c|c|c|}
\hline $\begin{array}{c}\text { Coordination } \\
\text { Game }\end{array}$ & & $\begin{array}{c}\text { Column } \\
L\end{array}$ & Player \\
\hline Row & L & $(0,0)$ & $(-1,-5)$ \\
\hline Player & $\mathrm{H}$ & $(-5,-1)$ & $(3,3)$ \\
\hline
\end{tabular}

Figure 2

For the game in Figure 2 both $(L, L)$ and $(H, H)$ are pure strategy Nash equilibria for the one-shot coordination game. We can denote the outcome from the Prisoners' Dilema game as $\gamma \in \Gamma$ where $\Gamma=\{(C, C),(C, D),(D, C),(D, D)\}$ is the set of all possible pure strategy plays. A pure strategy for the two stage game can be written as follows. For the row player, $s=\{s, f\}$, where $s \epsilon$ 
$\{C, D\}$ and $f: \Gamma-\{L, H\}$. For the column player, $T=(t, g)$, where $t$ $\epsilon\{C, D\}$ and $g: \Gamma \rightarrow\{L, H\}$.

By adding the coordination game the threat of a coordination failure in stage two can serve to discipline non-cooperative behavior in the Prisoners' Dilemma game. Before playing the two stage game suppose both players both agree to pick $s=t=c$ in stage one, and if $(C, C)$ is observed to play $f$ in stage two, i.e., $f((c ; c))=g((C, C))=H:$ otherwise, if anything else is observed in stage one they agree to play $I$ in stage two, i.e., $f(\gamma)=g(\gamma)$ $=$ I for all $\gamma \in\{(C, D),(D, C),(D, D)\}$. Notice this results in a total payof of 6 for each player. The attempt to defect against cooperation earns more in stage one but less in stage two. Payoffs have been constructed so that the best either player can do by defection is earn 5. The pre-play agreement to cooperate is self-enforcing. This example also demonstrates ore of the problems that emerges in understanding cooperation in repeated games. There are many equilibria for the two stage game where cooperation in the Prisoner's Dilemma is only one possibility. Any combination of Nash equilibria of the individual stage games will be a Nash equilibrium for the two-stage game. For example, $s=t=D$ in stage one, and $f(\gamma)=g(\gamma)=L$ for all $\gamma \in \Gamma$.

In the example above our. choice of payoffs allows players' to achieve cooperation in two stages, but how robust are selfenforcing equilibria for arbitrary payoffs? For now we will limit this question to games of complete information. Suppose Row and Column repeatedly play only the Prisoners' Dilemma game a 
large but finite number of times, say N. They should each play $(D, D)$ at $N$ since this last game is a one shot game." If at any point in time, $t$, players know they will each choose, $D$, every period in the future, then their best response at time $t$ is to pick D. By backwards induction, cooperation cannot be achieved in any finite period of time. See selten (1975).

However, if we allow players to play an infinite number of times with preferences over infinite sequences of payoffs (defined by discounting the sum of payoffs), then Friedman (1971) shows that players can use trigger strategies to earn an average payof of $(3,3)$ for discount factors, close to 0 . In particular, let $\gamma^{t} \in \Gamma$ be the history of play at time $t$. A strategy for each player is a function $s_{1}$ defined on countably long histories defined as follows:

$$
S_{i}\left(\gamma^{0}, \ldots, \gamma^{t-1}\right)=S_{i}^{t} \varepsilon\{C, D\}
$$

Player I's payoff can then be defined by,

$$
R_{i}\left(S_{p}, S_{-t}\right)=(1-\delta) \sum_{t=1}^{\infty} \delta^{t-1} I I_{i}^{t}\left(s_{i}^{t}, s_{-i}^{t}\right),
$$

where $\delta$ is player i's discount factor and $I I_{1}^{t}\left(s_{i}^{\tau}, s_{1}^{t}\right)$ is i's payoff in the t'th stage game when $i$ chooses $s_{i}^{t}$ and $i$ 's counterpart chooses $s_{.1}^{t}$. At any time the trigger strategies give each player the payoff $3 /(1-\delta)$ as long as players continue to cooperate. A player who defects gets a payoff of 5 but zero 
from then on. So as long as $\delta>3 / 5$ players' trigger strategies constitute a Nash equilibriur.

The requirement that players be infinitely lived in order to cooperate in the Prisoners' Dilemma game is problematic. But a number of experiments (see Rapoport (1987) and Selten and stoecker (1986)) demonstrate that subjects cooperate in relatively short finite horizon play. This has led to the study of the role of reputations. in sustaining cooperation in games with incomplete information (see Harsanyi 1967). The basic idea is if players are uncertain about other players' types then the possibility emerges that players will mimic (develop a reputation as) a type different from their own. In circumstances where cooperation is mutually beneficial players have an incentive to mimic cooperative behavior.

The role of reputations in the Prisoners' Dilemma game is studied by Kreps, Milgrom, Roberts and Wilson (1982). Suppose for example that it is common knowledge that there is a 50-50 chance that row is a type of player that is constrained to play (o $=$ has appropriate incentives) to play tit-for-tat and that the game will be played three times. Furthermore, before the game begins row will learn his or her own type. We will call the two possible row types Rational and Tit-for-tat. We can now solve for the Nash equilibrium of this game using backwards induction. 
Period 3: This is the last play of the game. Both column and Rational row will defect since this is a dominant -strategy. Tit-for-tat will play whatever column played in period 2 .

Period 2: If column is sure of row's type, i.e., row played $D$ in period 1, then by backwards induction both row and column will play $D$ in period 2.

Rational row will play $D$ in period 2 since this is a dominant strategy given that both players will play $D$ in period 3 .

If column is still unsure of row's type and column played $C$ in period 1 , then

a. column's expected payoff from playing $C$ in period 2 (and then $D$ in period 3$)$ is .5(3+5)+.5 $(-2+$ $0)=3$, given that rational row plays $D$ in period 2.

b. column's expected payoff from playing $D$ in period 2 (and then $D$ in period 3) is .5(5+0)+.5(0) $=$ 2.5, again given, that rational row plays $D$ in period 2 .

Since $3>2.5$ column's best response is to play $C$ in period 2 (and hope he or she is playing Tit-for-tat). If column is unsure of row's type and column played $D$ in period 1 , then 
a. column's expected payoff from playing $c$ in period 2 (and then $D$ in period 3 ) is .5(-2+5)+.5(0+ 0) $=1.5$, given that Rational row plays $D$ in period 2 .

b. column's expected payoff from playing $D$ in period 2 (and then $D$ in period 3 ) is $.5(0)+.5(0)=0$, again given that Rational row plays $D$ in period 2 . Since $1 \% 5>0 \cdots c o l u m n ' s$ best response is to play $c$ in period 2 .

Period 1: If column plays $C$ in period 1, followed by $C$ in period two and $D$ in period 3, then column's expected payoff is,

$$
.5(3+3+5)+.5(3-2+0)=6 \text {, if rational row }
$$

plays $C$ in period 1 , and $.5(3+3+5)+.5(2+0)=$ 4.5, if rational row plays $D$ in period 1 . Note once rational row plays $D$, column knows row is rational. If column plays $D$ in period 1 , followed by $C$ in period two and $D$ in period 3, then column's expected payoff is, $.5(5-2+5)+.5(5-2+0)=5.5$, if rational row plays $C$ in period 1 , and $.5(5-2+5)+.5(0)=4$, if rational row plays $D$ in period 1 .

Since $6>5.5$ and $4.5>4$ column should choose $c$ in period 1 .

If rational row chooses $C$ in period 1 , followed by $D$ in periods 2 and 3 , then rational row earns $(3+5+0)=8$. If rational row chooses $D$ in period 1 , followed by $D$ in : 
periods 2 and 3 , then rational row earns $(5+0+0)=5$. Again, note that by playing $D$ in period 1 rational row gives away his type and column will play $D$ in period 2 and 3. Since $8>5$, rational row will play $C$.

In conclusion, column will play (C, C, D), tit-for-tat row will play (C, C, C), and rational row will play (C, D, D). Expected payoffs are 6, 4, and 8 respectively.

Kreps, Milgrom, Roberts and Wilson show that, even when the probability of tit-for-tat type is very small, there exist conditions such that there will still be cooperation in finite, but longer, time. In their examples, players cooperate from the beginning until near the end of the game, and then defect. In summary, the theory of repeated games helps to explain how rational players can overcome myopic (stage-game) self-interest in favor of cooperation. The strength of this approach is that $i t$ is still based on individual (but longer run) self-interest, and it is parsimonious. The weakness of this approach is that it admits many possible equilibria without suggesting why cooperation is the most likely outcome. Moreover, for reputation based equilibria, people must entertain beliefs about certain types. But where do these beliefs come from? The next section examines the possibility that types emerge from the evolutionary fitness of certain cognitive abilities which predispose people towards reciprocity. Actual circumstances and experiences may lead to reciprocal behavior by some persons. Not everyone has to be of this type, but the type must exist in sufficient numbers 
for people to learn to believe in such types. Once beliefs exist, reputations follow. From reputation comes the stuff of culture.

III. Mental Algorithms for Social Exchange: Strategies in Human Cognition that Support Cooperation

The complex organization of the human mind is known to be the product of at least a few million years of evolutionary adaptation to solve the problems of hunting and gathering. Evolutionary psychologists hypothesize that these problems were solved not only by biological and neurological change, but also by adaptations in human social cognition (see Cosmides and Tooby, 1992, hereafter CT, and the references therein). The idea is that humans have special and highly developed cognitive mechanisms for dealing with social exchange problems. These cognitive mechanisms are referred to as cooperation, reciprocal altruism or simply reciprocity. Evolutionary psychologists argue that mental modules for solving social problems are as much a part of the adapted mind as our vision and hearing-balance faculties. ${ }^{2}$ Evolutionary biologists consider adaptations to be cellular, neurophysiological, chemical, and other mechanisms that solve specialized design problems.

Some diverse examples of the study of mental "computational" modules that solve specialized design problems include: vision, language and "mind reading." The mechanism which constitutes vision involves neural circuits whose design solves the problem of scene analysis (Marr, 1982). The solution to this problem employs specialized computational machinery for detecting shape, 
edges, motion, bugs "(in frogs), hawks (in rabbits), faces, etc,. Just as we learn by exposure, to see and interpret scenes Without being taught, we learn to speak without formal training of any kind.

Although "culture" is known to operate on our mental circuitry for language learning, the deep structure of language is common across cultures (Pinker, 1994). Normal English speaking preschoolers can-apply mental algorithms to root words to form regular noun plurals by adding 's' (Pinker, 1994, pp. 423), and the past tense of regular verbs by adding 'ed.' The preschooler even 'knows' that you can say that a house is miceinfested but never that it is rats-infested, that there can be teethmarks but never clawsmarks - the mental algorithm here allows compound words to be formed out of irregular plurals but never out of regular plurals. This is because of the way the brain works: regular plurals are not stem words stored in the mental inventory, but words derived algorithmically by the inflectional rule to add 's'. Preschoolers automatically make this distinction (Pinker, 1994, p. 146-7). That the mind contains blueprints for grammatical rules is further indicated by a language disorder in families which appears to be inherited like a pedigree with a dominant gene. Those afflicted with this disorder are unable to inflect root words to form derivatives such as the English 's' rule for obtaining plurals.

"Mind reading" - the process of inferring the mental states of others from their words and actions - facilitates "social 
understanding, behavioral predictions, social interaction, and communication" (Baron-Cohen, 1995, p. 30). Autism in children makes them mind blind - they are not automatically aware of mental phenomena, and cannot "mind read" (Baron-Cohen, 1995). A genetic basis is suggested by its greater risk in identical twins and biologically related siblings. Baron-Cohen (1995, pp. 88-95) implicates the amygdala and related areas of the brain as jointly controlling the ability to detect eye direction in others and to interpret mental states (have a theory of mind) for others. If our minds are also predisposed to learn behavioral responses that promote cooperative outcomes, CT argue that this should be detectable through controlled experiments designed to test specific hypotheses about such behavior. This doesn't mean that we are born with such capability; we only need to be born with the capacity to learn it developmentally from social exposure, much as we are born with the capacity to learn any language, but not with the ability to speak. A capacity for the natural learning of strategies that induce cooperation in social exchange has fitness value. But the particular form of what is learned can vary widely, depending upon the environment, accidents of nature, and how parental, familial, and societal units organize exchange processes. Consequently, "culture" is endlessly variable, but, functionally, reciprocity is universal.

In this section we focus on the experiments that have been used to investigate mental algorithms for social exchange. In the next few sections we explore how these results relate to what 
we have learned from a large variety of bargaining and market experiments.

Natural selection promotes the evolution of strategies for fitness in social exchange. These strategies are hypothesized to be embodied in the designs that modulate reasoning about social exchange. An analysis of these strategies allows one to deduce the behavioral characteristics of the associated mental algorithms. This analysis also allows predictions about human responses in reasoning experiments of the kind that we discuss below. These psychology experiments are of particular interest to experimental economists because of their obvious complementary relationship to subject behavior in games of strategic interaction.

Consider again the Prisoner's Dilemma game shown in Figure 1, but think of the entries corresponding to C (cooperate) or D (defect) for the row and column players as net benefits and net costs measured in units that increase (or decrease) the individual's inclusive fitness. C might represent the strategy "trade," while D might represent "steal." As discussed above, game theory predicts that mutual cooperation will not emerge in a single-move game.

Imagine a tournament which matches pairs from a large population of organisms so that the same two individuals are never matched a second time. Each member is matched one or a few times, reproduces itself, and dies. The offspring inherits the strategy choice propensity of the parent, and the number of 
offspring is proportional to the payoff gains of the parent in its matched plays of the game. Each generation repeats this process.

The models of repeated game interaction discussed in the previous section can be used to analyze equilibrium outcomes in such a game, since humans interact repeatedly. In fact, repeat interaction is a prominent characteristic of social exchange. Needs are rarely simultaneous. But long before human societies invented a generally accepted medium of exchange, various cultural mechanisms provided social adaptations which allowed delayed mutual benefits to be gained: I share my meat with you when I am lucky at the hunt, and you share yours with me when you are lucky. Although evolutionary biologists refer to this as reciprocal altruism, we prefer to call it reciprocity. I am not really being an altruist if my action is based on my expectation of your reciprocation.

Reciprocity leads naturally to property rights. If I grow corn and you grow pigs, and we exchange our surpluses, then we each have an interest in the other's property right in what is grown. If either of us plays "steal," that ends the trading relationship. Hence, mutual recognition and defense of informal property right systems needn't require the preexistence of a Leviathan.

But how might such mutual cooperation emerge in a repeated version of the game? Necessary conditions, as they are usually stated, follow from the previous discussion of conditions for 
cooperation in repeated games. There must be (i) a high probability that players will interact again, (ii) no end game problem (death is uncertain, heirs count, etc; otherwise cooperative play unravels from the end), and (iii) future payoffs are discounted relative to near payoffs, so that all players are impatient.

We know from the work of Axelrod and Hamilton (1981) that strategy C cannot be selected for in repeated play, but that the contingent cooperative strategy, $T$ (tit-for-tat), can be selected for. In general it can be shown that any strategy, including $T$, can successfully invade a population of defectors if (and only if) it cooperates with cooperators and punishes defectors (Axelrod, 1984). As noted by СT (1992, p. 176-7), it is an empirical issue to determine which strategy, out of this admissible set, is actually embodied in human cognitive programs.

CT 1 ist 14 design features that must characterize the mental algorithms of such a strategy for any organism.

(1) Ability to detect offers of an exchange and an expectation of reciprocation (we later call this signalling).

(2) Ability to estimate own costs and benefits of various actions.

(3) Ability to estimate others' costs and benefits of various actions for purposes of initiating an exchange.

(4) Ability to estimate the probabilities that these actions will occur in any case (i.e., in the absence of exchange you need to know or be able to find out about the opportunity cost of exchange).

(5) Ability to determine if the benefits exceed the costs of an exchange to decide whether to accept an exchange offer. 
(6) Ability to include responses that cause offers to be rejected when costs exceed benefits.

(7) Ability to cause acceptance when benefits exceed costs.

(8) Ability to detect the intercontingent features of an exchange.

(9) Ability to translate the exchange into the individual values accruing to each agent.

(10) Capability of detecting cheaters in order to punish them, and, we would add, a capability of detecting acts of reciprocity in order to reward them.

(11) Ability to invoke punishment of cheating when the circumstances are appropriate. Later we provide data to show that cheating is forgiven early, but it is not tolerated later, in repeat plays of an extensive form game.

(12) Ability to remember the history of previous exchanges to know when to cooperate, defect, or punish.

(13) Ability to recognize different individuals. Later we show that same-pairing yields high cooperation; but random pairing also yields cooperation, so well-formed are our mental algorithms for some applications.

(14) Finally, the algorithms need not detect people who are always altruists, since they will tend not to survive in the population in an evolutionary process.

We think some of these design characteristics are stronger than necessary, as will be seen below in the context of particular experimental game results. Moreover, the above list is not intended to be exhaustive or sufficiently detailed to account for the fine structure of certain specific human interactions. For example, the Ache of Paraguay share low variance food products from gathering only within the nuclear family; yet, they share the high variance products of hunting throughout the tribe (Kaplin and Hill, 1985). 
The need to solve the Prisoner's Dilemma problem in order to achieve cooperation provides an abstract schema for motivating some of the items in the above list of design characteristics, and for organizing our thoughts about cooperation beyond immediate kin. For example, direct punishment of cheaters can lead to cooperative outcomes in a repeated game with discounting. However, simply referring to the motivating example of the Prisoner's Dilemma will not carry us to a full understanding of human social exchange. In particular, such an analysis will not help us understand why humans may engage in cooperative behavior toward anonymous strangers when there is no repeat play.

An important question concerning the mental algorithms humans use in social exchange is whether they consist of a few content-free generalized rules of reasoning, or whether they consist of designs specialized for solving problems of cooperation in social exchange. Both cognitive psychology and economic/game theory are driven by the principle that humans use content-free generalized rules of reasoning in solving decision problems.

CT (1992) argue that the evolutionary perspective favors the specialized over the generalized rules. General rules, applicable to any subject matter will not allow one to detect cheaters ... because what counts as cheating does not map onto the definition of violation imposed by the propositional calculus. Suppose you and I agree to the following exchange: 'If you give me your watch then I'l 1 give you $\$ 20 . '$ You would 
have violated our agreement - you would have cheated me - if you had taken my $\$ 20$ but not given me your watch. But according to the rules of inference of the propositional calculus, the only way this rule can be falsified is by your giving me your watch but my not giving you \$20" (CT, 1992, p. 179-180). That is, the way you falsify "if $P$, then $Q$, statements is to look for "P, not Q," evidence.

Social exchange situations do not always follow such logical rules. Furthermore, rules for detecting cheaters won't work in the presence of bluffs and double crosses. Hence, CT (1992) make the inference that there is a need for a rich variety of contentspecific mental rules for effective social exchange.

The CT research program is to design experiments that will test these kinds of propositions. We summarize here enough of this program to show how it links up with various experimental economics results discussed below. In the interest of brevity we will describe the experimental paradigm used by CT (1992, pp. 181-206), summarize a few of the basic experiments, then outline the pattern of results and their relevance to social exchange contracts, leaving the remaining details for the interested reader to explore in the literature.

The selection task which CT employ was developed by Wason (1966), whose motivation was to inquire as to whether the ordinary learning experiences of people reflected Karl Popper's hypothesis testing logic. The logic of falsifying a statement of the form if $P$ then $Q$, requires people to understand that it is 
violated only under the condition that $P$ is true and $Q$ is false. The procedure for each specific description of $P$ and $Q$ is as follows." Four cards each carry one label of the form $P$, not-P, Q, not $Q$ on the side facing up, and another of the same four labels on the side facing down. Each card corresponds to a person with one of the labeled properties. The rule is violated only by a card that has $a$ on one side and a not-Q on the reverse side.

Subjects are asked to indicate only those card(s) that definitely need to be turned over in order to see if any cases violate the rule. The correct answer is to select the cards showing $P$ (to see if there is a not-Q on the other side) and not$Q$ (to see if there is a $P$ on the other side). Here is an example of the task when $i t$ is presented as an abstract problem.

CT $(1992$, p. 182).

Part of your new clerical job at the local high school is to make sure that student documents have been processed correctly. Your job is to make sure the documents conform to the following alphanumeric rule:

"If a person has a ' $D$ ' rating, then his document must be marked code ' 3 '."
( I f
$P \quad$ (D)
then
$Q(3)) *$

You suspect the secretary you replaced did not categorize the students' documents correctly. The cards below have information about the documents of four people who are enrolled at this high school. Each card represents one person. One side of a card tells a person's letter rating and the other side of the card tells that person's number code. 
Indicate only those card(s) you definitely need to turn over to see if the documents of any of these people violate this rule.

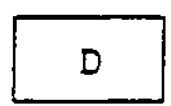

(?)

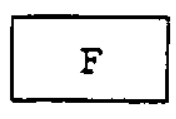

(not-P)

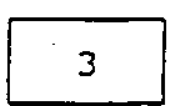

(Q)

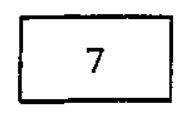

$($ not $-Q)$

*Parenthetical entries added to actual instructions. The correct responses are to indicate the cards showing the letter $D$ and the numeral 7 . The results of this experiment show that less than 25\% of college students choose both of these cards correctly.

Now consider an example that concerns a law which states that "If a person is drinking beer, then he must be over 20 years old." The correct response is to choose the card "drinking beer" and the card "I6 years old" (a third card reads "not drinking beer"; a fourth reads "25 years old"). In this experiment about 75\% of college students get it right. There are many differences between this example and the previous one, but note that one difference is that the second is a social contract rule.

Here are just a few of the many "descriptive" (non social contract) rules that have been tested since the 1960s:

(1) "If a person goes to Boston, then he takes the subway."

(2) "If a person eats hot chili peppers, then he will drink cold beer."

(3) "If you eat duiker meat, then you have found an ostrich eggshell."

(4) "If there is an 'A' on one side of a card, then there is $a$ ' $^{\prime}$ ' on the other side." 
Although people do better in more familiar examples such as going "to Boston," less than half get it right. A survey of this literature (Cosmides, 1985) suggests that "Robust and replicable content effects were found only for rules that related terms that are recognizable as benefits and cost/requirements in the format of a standard social contract ..." (CT, 1992, p. 183). Sixteen out of 16 experiments using social contracts showed large content effects. For non-social -contract rules, of 19 experiments 14 produced ho effect, 2 produced a Weak effect and 3 produced a substantial effect.

These findings launched a number of studies designed to separate the social contract hypothesis from confounding interpretations. The most obvious proposition needing further testing was that the results were driven by familiarity. The bottom line from several such studies was that "Familiarity cannot account for the pattern of reasoning elicited by social contract problems" (CT, 1992, p. 187).

In all of the social contract cases, the correct adaptive response is identical to the correct logical response. Hence, the social context may simply be facilitating Popperian reasoning. Can more crucial experiments be designed to separate this confounding effect? The evolutionary psychology literature has produced two distinct groups of experiments that confront this issue: (i) experiments which switch the $P$ and $Q$ in the standard social exchange exercise thereby breaking the identity between cheating detection and the correct logical response; 
(ii) experiments that change the subjects' perspective from own to other, thereby changing the interpretation of what constitutes cheating.

An example of a switched social contract is the following: Standard form: "If you give me your watch, I will give you $\$ 20 . "$ Switched form: "If I give you $\$ 20$, you give me your watch." I have cheated you if I accept your watch without paying $\$ 20$, but in switched form this corresponds to observing $Q$ and not-P, which is not the logically correct response. In switched form, an unfamiliar example would be (3) above, stated "If you have found an ostrich eggshell, then you eat duiker meat." In the standard form Wason experiments, not-P and $Q$ were rare responses; but in the switched experiments they were very common, e.g. $67 \%$ and $75 \%$ of the subjects. This corresponds to the predictions of adaptive social contract theory as described by CT (1992).

An example of the perspective change experiments uses the following statement: "If an employee gets a pension, then that employee must have worked for the firm for at least 10 years." In standard form the subject is the employer; in alternative form he or she is the employee. Hence, from the perspective of the employer cheating occurs when a pension goes to an employee who has not worked for the firm for at least 10 years ( $P$, and not-Q). From the perspective of an employee, cheating occurs when an employee has worked for at least 10 years, but fails to get a pension (not-P, and Q). These are the correct responses from adaptive social contract theory. But regardless of one's role 
the correct Popperian response is $P$ and not-Q: the employee gets the pension and worked for the firm less than 10 years. The experimental results strongly support adaptive theory: subjects predominantly choose $P$ and not-Q when they are the employer (70$80 \%)$, but not-P and $Q$ when they are the employee $(60-65 \%) .^{3}$

IV. Observability, Communication, and Forward Signalling If humans are preprogrammed to achieve cooperative outcomes in social exchange environments by identifying and punishing cheaters, then factors that facilitate the operation of these natural mechanisms should increase both cooperative behavior and outcomes even in the presence of contrary individual incentives. For example, cooperative behavior should increase if individuals can observe and monitor one anothers' behaviors, even if there are no direct mechanisms for enforcing specific behaviors. If it is possible for agents to directly punish cheating by other agents, cooperative behavior should increase even further. Similarly, if agents can communicate with one another, they can frame a group decision as a social exchange problem and activate natural inclinations to cooperate. Thus, in this framework, communication can increase cooperative behavior and outcomes even if there are no effective mechanisms for monitoring and punishing cheaters. This kind of communication is often referred to as "cheap talk." In the absence of direct communication, if agents can signal other agents of their 
intentions, they can create shared expectations of social exchange and cooperative behavior.

We proceed next to review a considerable body of experimental economics data that show how cooperation increases with communication, observability, opportunities to punish cheaters even at one's own expense, and with the ability to signal intentions without direct communication.

IV.A. Voluntary Contribution Experiments

The standard environment in which to study the operation of the free rider problem is the voluntary contribution mechanism (VCM), extensively studied by Isaac and Walker, and their coauthors (Isaac, McCue, and Plott, 1985; Isaac, Schmitz, and Walker, 1989; Isaac and Walker, 1988a,b; Isaac, Walker, and Thomas, 1984; Isaac, Walker, and Williams, 1991). In a VCM experiment, each subject is given a set of tokens at the beginning of each period of the experiment. The subject may invest tokens in an individual exchange, which returns a specified sum of money for each token invested, or a group exchange, which returns money to the subject as a function of the total contributions of all the subjects in the experiment. The subject may invest all tokens in the individual exchange, all tokens in the group exchange, or divide tokens between the two exchanges using any desired percentage distribution to each.

Typically, in VCM experiments, the individual incentives are designed to make strong free riding, or $\$ 0$ contributions to the 
group exchange, the dominant strategy for each subject. On the other hand, the highest joint payoff for all subjects is achieved when all subjects contribute 100\% of their tokens to the group exchange. Thus, the experiment is designed as a multiperson Prisoner's Dilemma: cooperative behavior yields the highest joint payoff, but individual incentives do not support cooperative behavior as a noncooperative equilibrium of the contribution game.

Isaac and Walker and their coauthors, as cited above, find that contributions to the group exchange are sensitive to differences in the rules of message exchange that are meaningful in light of our previous discussion of cognitive mechanisms for social exchange. With subject groups of 4 or 10 subjects, if subjects make contributions in private, if there is no identified target level of contributions, and if they do not communicate with one another at any time during the experiment, then contributions to the group exchange decline from about $40 \%$ of tokens in period 1 to about 10\% of tokens in period 10 (Isaac and Walker, 1988a; Isaac, Walker, and Thomas, 1984).

However, in the same experimental environment, if subjects can talk with one another for a short period before each decision, contributions to the group exchange quickly rise to almost $100 \%$ of tokens, even if actual investment decisions are made in private (Isaac and Walker, 1988b). This set of experimental results illustrates the importance of "cheap talk" communication in creating an environment in which agents expect 
one another to behave cooperatively; and they abide by the reinforced norm even when a 11 decisions are made in private and no individual's defection can be detected by the other participants.

These results can be interpreted in a signalling context. During the communication phase, individuals verbally signal that they will behave cooperatively and that they expect others to reciprocate by behaving cooperatively. During the decision making phase, individuals generally abide by the norm reinforced by the signal, and a cooperative outcome is achieved. While no direct punishment can be inflicted by other subjects in the event of defection, other subjects can exact general punishment by defection against other subjects in future rounds. This suggest that some of CT's design characteristics, such as 10,11 ana 13 can be weakened.

In another set of experiments in this series (Isaac, Schmitz, and Walker, 1989), the experimenter establishes a minimum level of contribution (called a provision point) before any group investments yield returns. Comparing results with a provision point to results without a provision point, allowing no communication in either set of experiments, contributions to the group account increase significantly in the presence of a provision point. If the provision point is set at $100 \%$ of tokens, contributions rise even further, although many groups fail to attain the $100 \%$ provision point. 
From a signaliing prospective, the provision point signals to subjects an expected joint level of contribution to the group account: i.e., the provision point helps to induce common expectations of substantial contributions to the group account. If all subjects are endowed with the same number of tokens each period there is an implied signal that each subject should contribute $(1 / n)$ P of his or her tokens to the group account in order to achieve the provision point, where $\mathrm{P}$ is the rumber of tokens required to attain the provision point.

IV.B.' UItimatum and Dictator Experiments

Ultimatum and dictator experiments illustrate the importance of observability, shared expectations of social norms, punishment, and signaliing in enforcing sharing behavior. In an ultimatum game, player 1 makes an offer to player 2 of $\$ x$ irom $\equiv$ total of $\$ M$. If player 2 accepts the offer, then player 1 is paid $\$(M-X)$ and player 2 receives $\$ x$; if player 2 rejects the. offer, each gets $\$ 0$. In the dictator game, player 2 must accept player 1's offer.

If we assume subjects come to an ultimatum or dictator game with no preconceived norms of behavior in such an envizonment, that all subjects prefer more money to less, and that ali subjects have common knowledge of these characteristics of all other subjects, then the noncooperative equilibrium of the ultimatum game is for player 1 to offer player 2 the swallest $\$$ unit of account, and for player 2 to accept the offer. In the 
dictator game player 1 offers $\$ 0$ to player 2 . In the ultimatum game, however, player 2 can punish player 1 for "cheating" on an implied social norm of sharing across time, learned in social exchange experience, by rejecting player 1's offer. That response is a dominated strategy, if viewed in isolation, since both players would be financially better off even with a vanishingly small offer. But, in the absence of common knowledge, the possibility of punishment may change player l's equilibrium strategy. The dictator game is essentially equivalent to the ultimatum game, except that no opportunity for punishment is provided.

In Kahneman, Knetsch, and Thaler (1986) (hereinafter KKT), players 1 and 2 in an ultimatum game are "provisionally allocated" $\$ 10$ and player 1 is asked to make an initial offer to "divide" the $\$ 10$ between the two players. player 2 may veto the division, in which case they both get $\$ 0$. There is no direct contact or communication between the players and they only play the game once. KKT find that most player 1's offer $\$ 5$ to player $2 ' s$; offers less than $\$ 5$ are sometimes rejected. The general features of these results have been replicated in cross cultural comparisons suggesting that the results are not culture-specific "(Roth,""Prasrikar, Okuno-Fujimara and Zamir,"1991).

Forsythe, Horowtiz, Savin, and Sefton (1994) (hereinafter FHSS) replicate KKT's results from the ultimatum game, and also study the dictator game. They find that about $20 \%$ of dictator player is offer $\$ 0$ to their player 2 counterparts, as 
noncooperative game theory would predict; however more player is offer $\$ 5$ than offer $\$ 0$, and offers of $\$ 1, \$ 2, \quad \$ 3$, and $\$ 4$ are approximately evenly distributed. Thus, removing the threat of punishment reduces sharing behavior, but not by as much as noncooperative game theory predicts.

Recognizing that the prospect of punishment might create expectations that change player 1's behavior, Hoffman, McCabe, Shachat, and Smith (1994) (hereinafter HMSS) consider experimental treatments explicitly designed to affect subject expectations about operating norms of social exchange. The experimental instructions that describe the different treatments might be viewed as forward signals to the subjects of the expected social norm operating in each experiment.

Brewer and Crano (1994), in a recent social psychology textbook, identify three important norms of social exchange that may apply in ultimatum games. The norm of equality implies that gains should be shared equally in the absence of any objective differences between individuals that would suggest some other sharing rule. The norm of equity implies that individuals who contribute more to a social exchange should gain a larger share of the returns. The norm of reciprocity implies that if one individual offers a share to another individual, the second individual is expected to reciprocate as soon as possible. In our discussions to follow we will distinguish negative reciprocity - the use of punishment strategies to retaliate against behavior that is deemed inappropriate - and positive 
reciprocity - the use of strategies that reward appropriate behavior.

The des,ign of KKT and FHSS invoke the norm of equality. No distinction is made between the two individuals "provisionally allocated" $\$ 10$ and they are told to "divide" the money. Not surprisingly, perhaps, in such an environment, deviations from equal division are punished as "cheating'' on the implied social exchange. HMSS replicate the KKT and FHSS results in a slightly different experimental environment. The key elements they maintain are: (1) players 1 and 2 are randomly assigned to those positions; and (2) the task is described as proposing a "division" of $\$ 10$ "provisionally allocated to each pair." They refer to this treatment as random/divide $\$ 10$.

To invoke the norm of equity, HMSS explore two treatment changes in a $2 \times 2$ experimental design. First, without changing the reduced form of the game, HMSS describe it as a market in which the "seller" (player 1) chooses a price (division of \$10) and the "buyer" (player 2) indicates whether he or she will buy or not buy (accept or not accept). From the perspective of social exchange, a seller might equitably earn a higher return than a buyer. Treatments with buyers and sellers are referred to as exchange treatments.

Second, they make the sellers earn the right to be a seller by scoring higher on a general knowledge quiz than buyers. Winners are then told they have "earned the right" to be sellers. Going back to Homans (1967), social exchange equity theory 
predicts that individuals who have earned the right to a higher return will be socially justified in receiving that higher"

return. Treatments using a general knowledge quiz to assign property rights in the seller's first-mover position are referred to as "contest'' treatments.

Figure 3 reproduces HMSS's random/divide and contest/exchange experimental results. As social exchange theory would predict, in a situation in which it is equitable for player 1 to receive a larger compensation than player 2 (i.e., contest/exchange) player 1 offers significantly less to player 2, and player 2 accepts with the same probability (no significant difference in the rejection frequencies). These results suggest that the change from random/divide to contest/exchange alters the shared expectations of the two players regarding the social exchange norm operating to determine an appropriate sharing rule. Moreover, Hoffman, McCabe, and Smith (1995a) (hereinafter HMSa) find no difference when the amount to be divided is increased from $\$ 10$ to $\$ 100$ in these two treatments. This result suggests that the norms of social exchange are very strong; as the stakes are increased, so is the opportunity cost of proposing an improper division and getting punished. Finally, the difference between random/divide and contest/exchange carries over to a dictator experiment as well. Thus, the change in expectations takes place even when there is no threat of punishment from player 2 . 
But why do these treatments alter the expectations of both players coincidentally? Offers are reduced without causing an increase in the rejection rate. This, we would suggest, appears to be a manifestation of the human ability to infer mental states - in this case expectations - from relevant information. "Mind reading" also implies the ability to take the perspective of another person with common" information. Obviously, this can involve errors of perception, but here is a case where such errors are minimal."

The sensitivity of ultimatum results to small changes in the wording of instructions that relate to expectations of possible punishment for deviations from norms of social exchange is illustrated with a new, previously unpublished, data set. In this new experiment we replicate the random/exchange and contest/exchange treatments reported in HMSS with one small change in the sellers' choice forms. The following two sentences are added: "Before making your choice, consider what choice you expect the buyer to make. Also consider what you think the buyer expects you to choose." Otherwise the instructions and experimental procedures are identical to those reported in HMSS. Note that this change (ostensibly benign) "merely" calls the seller's explicit attention to the strategic feature of the interaction, including its punishment possibilities.

Figure 4 compares the experimental results, with and without the added instructions. Notice that the added instructions, asking sellers to think ahead about buyer responses, shift both 
the random and the contest offers back towards the more equal splits characteristic of KKT, FHSS, and the random/divide treatment reported in HMSS and reproduced in Figure 3. The random/exchange offers shift from a dual mode of $\$ 3$ and $\$ 4$ to a strong mode of $\$ 5$. In the contest/exchange experiments, the added instructions eliminate all offers of $\$ 1$ or $\$ 2$, and increase the proportion of offers between $\$ 4$ and $\$ 6$. Tables 1 and 2 show these shifts are significant under both the Epps-singleton test that tests for changes in the overall distribution, and the Wilcoxon test that tests for shifts in the distribution. These results are consistent with the hypotheses that the added instruction triggers the operation of a different mental module. The unguarded responses in the original experiments are now replaced by a more guarded strategy-based decision module that anticipates rejection if the offers are insufficiently generous. This module's response is the opposite of the domain-general content-independent game theoretic model of subgame perfect equilibrium. When subjects are exhorted to think about what your counterpart is likely to do, and what he/she expects you to do, the response is not "my counterpart's interest is to accept one dollar, therefore but rather "my counterpart may veto my offer unless it is generous."

Returning to the dictator experiments first outlined in FHSS, observability is a powerful component of the enforcement of social norms. In order for others to punish deviations from behavior in accord with social norms, such behavior must be 
observable. FHSS recognized this problem in designing their dictator experiments: player 1's and player 2's were recruited to separate rooms and had no contact with one another. Thus, all player 1 decisions were anonymous with respect to player 2. However, Player 1 decisions were not anonymous with respect to the experimenter. Someone was still "watching;" hence player 1's were still not entirely removed from abnormal social exchange setting where reciprocity norms of behavior might still apply.

HMSS design a new version of the dictator experiment, which they refer to as "double-blind." This experiment has several important characteristics that are later changed one or two at a time to investigate the role of social isolation in extinguishing behavior in accord with social norms (Hoffman, McCabe, and Smith, 1995b; hereinafter HMSb). (i) 15 subjects are recruited to Room A (player 1's) and 14 subjects are recruited to Room B (player 2's). One subject in Room $A$ is selected as the participant monitor and paid $\$ 10$. (ii) In Room $A$ there are 14 opaque envelopes in a box. Twelve contain 10 \$1 bills and 10 white slips of paper. Two contain 20 white slips of paper, making it impossible to detect which subjects might take a 11 the money, even if all who get envelopes containing money do so. (iii) One at a time, each non-monitor subject in Room A takes his or her belongings, selects an envelope, sits behind a large cardboard box at the back of the room to ensure privacy, (iv) removes 10 slips of paper which can be any combination of $\$ 1$ bills and white slips of paper, (v) seals the envelope, drops it in another box, 
and leaves the experiment. (vi) When a 1114 subjects have left Room $A$,"the monitor takes the box of envelopes to Room $B$ and $s$ its outside the door. (vii) Each subject in Room B is called individually by the monitor, who randomly selects an envelope, opens $i t$, records the number of $\$ 1 \mathrm{bills}$, and hands the envelope and any $\$ 1$ bills in it to the subject, who leaves the experiment. .In, this experiment, $64 \%$ of the player is leave $\$ 0$ for their corresponding player 2s; about $90 \%$ leave no more than $\$ 2$. These results are strikingly different from the dictator results summarized in FHSS, and from the HMSS random/divide and contest/exchange dictator experiments in which subjects were observed by the experimenters. HMSb then vary each of the elements of the double-blind dictator experiment in ways intended to reduce the degree of "social distance" between the experimenter and others who might see the data, and the subjects in Room A, while preserving complete anonymity between subjects in Room A and those in Room B. First, they remove (i) the participant monitor and ( $i$ i) the 2 envelopes containing no $\$ 1$ bills. Now, the experimenter comes in contact with the subjects; and they have no protection from detection if all subjects leave $\$ 0$ for their counterparts in Room B. They refer to this treatment as. double-blind2. In the next series of experiments, the experimenter opens the envelope and counts the $\$ 1$ bills left in $i t$ before each Player 1 leaves Room $A$. They refer to this treatment as single-blindl. Finally, the $\$ 1$ bills are replaced by a form which each player 1 fills out. The 
experimenter - at the heart of the transaction - looks at the form and pays each Player 1 the correct number of $\$ 1$ bills ( single-blind2).

Comparing the results of these experiments, and their replication of FHSS, and a variation of FHSS in which they remove the wording about "provisionally allocating" and "dividing $\$ 10, "$ they find that the experimental results form a predicted ordered set of distributions. As the social distance between the subject and others increases (decreases) the cumulative distribution of offers to Player 2's decreases (increases). These results demonstrate quite strongly the power of observability in enforcing social norms of equity and (implied) reciprocity.

IV.C. Signalling, Trust, and Punishment in Bargaining Experiments The experimental data summarized thus far suggest

consistently that experimental environments that signal the norms of social exchange situations, however subtle, to subjects, or allow some coordination through communication, lead to outcomes that are more cooperative (involve more explicit sharing) than noncooperative game theory would predict. On the other hand, experimental environments that isolate subjects from any social exchange context tend to result in subject behavior more in accord with single play game theory. In this section we review the results of 2-person extensive form bargaining experiments in which subjects move sequentially, and one subject can signal an intention to cooperate by making a move that is suboptimal at the 
particular point in the game the move is made, but which can lead the pair of subjects to higher joint payoff in the end. In some of these experiments, the signalling player, at a cost to himself or herself, can directly punish the other player for "cheating" on the implied social exchange. In other 'trust' experiments there is no direct opportunity to retaliate against defection from

a signal to cooperate.

IV.C.1 The Constituent Games: Payoffs

Figure 5 shows an extensive form bargaining tree for two constituent, or stage, games played by two persons. Player 1 begins with a move right or down at node $x_{1}$. A move right terminates the play with payoffs $(35,70)$, in cents per play (multiplied by 20 in single play) respectively for players 1 arid 2. If the move is down, then player 2 moves left or right at node $x_{2}$, and so on. Play ends with any move that terminates at a payoff box on the right or the left of the tree. Game 1 shows the basic payoff structure used; Game 2 is the same except for the payoffs in the boxes corresponding to plays left at nodes $\mathbf{x}_{3}$ and $\mathbf{x}_{5}$. McCabe, Rassenti and Smith (1994) have studied behavior in these games under a variety of matching protocols and information treatments.

In both Games 1 and 2 the right side of the tree contains the subgame perfect (SP) noncooperative outcome $(40,40)$, where Player 2 moves right at $\mathbf{x}_{6}$. This outcome is achieved by simple dominance, once Player 2 moves right at $x_{2}$; $i . e .$, it is in player 
I's interest to play down at $x_{4}$, and for Player 2 then to play right at $x_{6} \cdot 5$

In Game 1 cooperative actions by the players can lead to the largest symmetric (IS) outcome, achieved if player I moves left at $x_{3}$. Under complete payoff information, a move left at $x_{2}$ by Player 2 can be interpreted as a signal to player 1 that player 1 should go"left at $x_{3}$ fThis is because 50 at IS is clearly better than 40 at SP for Player 2, allowing Player 1 to infer Player 2 's reason for playing left at $x_{2} \cdot$ ) Player 1 , however, can defect, move down at $x_{3}$, and force Player 2 , in his or her own interest, to move left at $x_{5}$ giving Player 1 a payoff of 60 . In fact this is the game theoretic prediction if play occurs on the left side of the tree in Game 1. In a single play, player 2 should see this and the theoretical prediction becomes selten's SP outcome on the right.

But a move left at $x_{2}$ in Game $l$ is more than a signal that Player 2 wants to achieve the LS outcome $(50,50)$. It can also be interpreted as coupling a signal with a potential threat to play down at $x_{5}$, punishing Player 1 if Player 1 defects or 'cheats' by playing down at $x_{3}$. This action, however, is costly to Player 2, since each player gets 20 if Player 1 moves left at $x_{7} \cdot 6$ Hence, even in a single play, given our discussion in the previous sections, it is not a good bet that the game theoretic outcome at SP will dominate. In repeated play the prospects for achieving the Ls outcome are of course much better. 
Game 2 contrasts with Game 1 in that to achieve LS, by Player 2 moving left at $\mathbf{x}_{5}$, Player 1 must resist the temptation to move left at $\mathbf{x}_{3}$. In Game 2, Player 1 can 'cheat' on the invitation to cooperate by choosing $(60,30)$ without the prospect that Player 2 can punish Player 1. Thus, Game 2 allows signalling, but not punishment; it is a game of trust.

IV.C.2 Matching Protocols and Information

Table 3 shows nine treatments that vary the protocol for, matching pairs, and subject information on payoffs, in each experiment. Except in the SINGLE treatments the game is played repeatedly for 20 trials using inexperienced subjects who are not informed as to when the last trial will occur, but who expect the game to be repeated much longer. This is made credible because, when the subjects are recruited, it is emphasized that they are volunteering for a two-hour session; yet, the experiment normally takes at most one hour and 15 minutes, including about 15 minutes for the instructions. An experiment consists of groups of approximately 12 subjects who are randomly assigned to six pairs.

The treatment RANDOM refers to the condition that in each play of the game subjects are randomly assigned a role (Player 1 or Player 2) and a counterpart. SAME is the treatment in which subject pairs and player roles remain the same for all 20 plays in a session. In REPEAT SINGLE we begin the session with 16 subjects, and each person plays every other counterpart once, with their roles alternating between player 1 and 2 . Under 
CONTINGENT each player'indicates a choice at each of his/her nodes. Then the computer executes the play. SINGLE means that all pairs play the constituent game exactly once for a multiple of 20 times the payoffs shown in the boxes of Figure 6 . These different matching protocols control the probability that a given subject will be matched with the same person on any two plays of the game...... Under SAME this probability is one; under SINGLE and REPEAT SINGLE it is zero. In RANDOM this probability is within the interval $[0,1]$.

We turn next to a summary of the research questions and the experimental results. Table 4 lists the conditional outcome frequencies for each payoff box. Reading across row 1 for SINGLE I we observe that of 26 pairs none ended at $(35,70)$; 13 of 26 Player 2 s moved Left at $x_{2}$ signalling cooperation; 10 of the 13 left plays ended with Player 1 choosing $(50,50) ; 3$ player Is defected by playing down at $x_{3} ; 2$ of these player 2 s accepted the defection and responded with $(60,30)$, while one played down at $x_{5}$ to punish Player 1 who then chose $(20,20)$. On the right game, played by 13 of 26 player 25,12 of 13 ended at the SP outcome $(40,40)$; one play was at $(15,30)$. The column labeled $E\left(\pi_{2}\right.$ 'Left) computes the expected profit, 44.6 cents, to player 2 from playing left at $x_{2}$, based on the relative frequencies of subsequent play by both players." $E(\pi, \mid$ Down) is the expected profit, 46.7 cents, to Player 1 from defecting at node $x_{3}$. Efficiency is the percentage of the cooperative total payoff at $(50,50)$ that is realized by all players. Thus in SINGLE $185.5 \%$ 
of the cooperative surplus is collected by all pairs. At SP efficiency is $80 \%$, so any greater efficiency implies a net efficiency benefit from cooperative initiatives.

IV.C.3 Summary of Results

Result 1. Complete information game theory predicts that in SINGLE 1 all plays will be in the right subgame.' In fact half are in the left subgame. In REPEAT SINGLE 1, we observe that experience does not help; now 58\% play the left subgame. In SINGLE 1 EXPERIENCED 17 subjects from RANDOM 1 return for a single play. Now $76.5 \%$ play the left subgame - in fact the play is more like $i t$ was another round in RANDOM 1 . Contrary to the theory we observe both too much attempted cooperation and too few defections on these attempts.

Result 2. Player $2 \mathrm{~s}$ who self select to play in the right subgame almost always end at the SP, $(40,40)$, as predicted by game theory. This is indicated by the high conditional probability of the SP outcome across all treatment conditions. Thus, SP is relevant for a subset of subjects who, for whatever reason, avoid the strategic hazards in the left subgame of both Games 1 and 2 .

Result 3. In all treatments it is (weakly) advantageous in the expected payoff sense to play in the left subgame. This is indicated by the fact that the expected profit to player 2 of 
left play is at least 40.0 cents in all treatments, and 40 , is the payoff to Player 2 at SP. Thus, the timidity of the minority who play the right subgame is unprofitable in both Games 1 and 2 .

Result 4. Defections by Player 1 at node $x_{3}$ of Game 1 are not profitable under any treatment: the expected profit of playing down is always less than 60 the payoff to player 1 if Player 2 forgoes the punishment option and plays left at $\mathbf{x}_{5}$. Thus, the "punish cheaters" mental module hypothesized by Cosmides (1985) is alive, well and used extensively enough to be effective, but not so much that efficiency is reduced.

Result 5. SINGLE 1 CONTINGENT converts Game 1 from the extensive to the normal form by requiring each player's choices at a 11 nodes of the tree to be made in advance for simultaneous play. It is equivalent to expressing all payoff path outcomes in matrix form for simultaneous choice by both players. Game theory hypothesizes that the normal and extensive forms are equivalent, but previous research has shown that this is not generally the case (Schotter, Wiegelt and Wilson, 1994). Comparing SINGLE 1 with SINGLE 1 CONTINGENT we see that left play declines (right play increases) in the latter. Our explanation is as follows: the extensive form, with sequential turn-taking moves allows the players to engage in a move interpreting conversation. Thus, at node. $\mathbf{x}_{2}$, Player 2 has just received the message, "I moved down at $\mathbf{x}$, because I want to do better than receive 35," from player 1 . If Player 2 now moves left, the message is "I am playing left 
because I want to forgo the $(40,40)$ on the right in favor of (50, 50) which is better for both of us. Also, note that if you respond by playing down at $x_{3}$, then $I$ have the option of punishing you with $(20,20)$." This potential dialogue is disrupted with simultaneous play, although under strict rationality it is irrelevant. That is, Player $2 s$ message is not credibly self enforcing. But of course real people infer mental states from actions and, as shown by these results, may play differently in the extensive form them in the normal form.

Result 6. When six pairs are randomly rematched and reassigned the role of Player 1 or 2 for 20 trials with unknown end game, the frequency of left play increases from $50 \%$ to $67.3 \%$ (cf SINGLE 1 and RANDOM 1 in Table 4). Subjects can now form expectations about the response behavior of members of the 12 person population in which they are interacting. They can learn (or sense), for example, that left play has a higher expected return than right play -44.6 cents $v 40$ cents in SINGLE 1 . But across repeated plays, there is slippage, with the expected profit from left play falling to an average of 40.7 cents. This is because attaining (50, 50) requires an increase in the overall levels of punishment. Thus more player $2 \mathrm{~s}$ learn that it pays to move left at $\mathbf{x}_{2}$, but, simultaneously, that if the punishment option is to be credible, its use must be increased: the conditional probability of $(50,50)$ declines from $76.9 \%$ in SINGLE 1 to $67.8 \%$ in RANDOM 1 , as defections rise. 
Result 7. Consistent with the game theoretic folk theorem that repeat interactions (can) promote cooperation by allowing reputations to form, left play increases from $67.3 \%$ in RANDOM 1 to $81.5 \%$ in SAME 1, and the realization of $(50,50)$ increases from $67.8 \%$ to $88.4 \%$; this allows the expected return from left play for player 2 to increase from 40.7 cents to 46.6 cents.

Result 8. The failure of the SP predicted outcome (Result 1) motivated the study of Game 2 in which the cooperative (50, 50) outcome cannot be supported by the prospect of punishment. Comparing SINGLE 2 with SINGLE 1 (rows 2 and 1 of Table 4 ), we see a slight, statistically insignificant, reduction in left moves by Player $2 \mathrm{~s}$ in Game 2. Play in left subgame 2 produces fewer $(50,50)$ outcomes (50\%) than in Game 1 (76.9\%). This reduces the expected profit of left play from 44.6 cents in Game 1 to a break-even 40 cents in Game 2. Clearly, the strategic differences between the two games, as implied by game theory, is making a difference in the predicted direction. But the more Interesting observation is that the trust element in Game 2 is sufficient to yield cooperation for half of the pairs who play the left subgame. This is consistent with results reported by Fehr Kirchsteiger and Riedl (1993). in labor market experiments, and by Berg, Dickhaut. and McCabe (1994) in investment dictator games. In both these studies first movers trusted second movers to reciprocate with no possibility of punishment. 
Result 9. Comparing RANDOM 1 and 2, left play declines strongly from $67.3 \%$ to $35.3 \%$ Without the prospect of direct punishment Player $2 \mathrm{~s}$ are much more reluctant to signal cooperation, although it actually pays (41.2 cents).

Result 10. Comparing SAME 1 and 2, left play declines less precipitously from $81.5 \%$ to $61.7 \%$, as the return to left play remains high $(46.9$ cents). Thus when the pairs remain constant, trust can develop, and cooperation is less dependent on the availability of a direct punishment option. Also, punishment by playing right on the next round is feasible with same pairing.

V. When Do People Abandon their Automatic Responses and Approximate Game-Theoretic Equilibria?

The above examples illustrate a model of the individual

which is a composite of a person whose play reflects some game theoretic principles, and some learned or innate responses involving signalling, trust, punishment and other ingredients of reciprocity behavior. This mixture of play objectives serves the typical subject group very well: they exceed the performance of strict game-theoretic players in that surplus improving cooperative outcomes are more often attained than theory would predict.

In this section we briefly summarize three experimental research programs in which subjects begin with their intuitive automatic responses, discover that these responses cannot, sustain good performance, then adjust in the direction of the rational 
expectations outcome predicted by theory. In each case subjects are given common information, but this is not sufficient to induce common knowledge in the sense of expectations. This, we argue, is because common information leaves behavioral or strategic uncertainty unresolved. The latter is resolved over time as subjects, in successive extensive form rounds, come to have common expectations that predicted equilibrium outcomes will prevail.

Smith, Suchanek and Williams (1988) report asset trading experiments over fifteen period horizons in which fundamental dividend value each period is common information. Muthian rational expectations theory predicts that trading, if it occurs, will be at prices near the declining path of dividend value. Dozens of experiments have documented the tendency of inexperienced subjects to produce bell-shaped price bubbles relative to dividend value (See Porter and Smith, 1995a for a summary). If the same group is brought back a second time, the bubble is dampened, and trading volume is reduced. If a third session is run with the same subjects, prices are near fundamental value with very low trading volume. Consequently, with experience across successive 15-period trading sessions, subjects come to have common rational expectations.

McCabe (1989) reports similar phenomena in a 6 period extensive form game using fiat money. In successive periods subjects use buy, sell and null messages to trade, or not, a unit of fiat money against dividend paying bonds. In the last, period 
a bond holder should not sell since he or she is left with worthless flat money. Similarly, the money should not be accepted on the penultimate round, and, by backward induction should not be accepted in the first period. Although subjects have complete information on this payoff structure, trade in the first play of the sequence yields trade in each period until the last one. Repeating this constituent, game for ten rounds (common information) causes some, but not a complete, unravelling backward from the final trial. When subjects return for a second 15 trial experiment, the slow unravelling process continues, but trade persists especially in the early rounds. In a third session for 20 trials, trade is further diminished, and is virtually eliminated by the 15th trial.

These results can be understood in terms of a model in which people have been strongly conditioned by reciprocity experience to accept fiat money in trade because they expect others to accept money when they offer i $t$ in trade. This expectation is unconscious; they never ask themselves why they and others accept money. It is a learned reciprocity response, which serves them effectively in daily life. They are recruited to the laboratory where the conditions for ongoing repeated exchange are not satisfied; in the end-game intrinsically worthless money is refused in trade. This failure experience induces them to reevaluate their unconscious, accustomed response to money. Very slowly, in the limit, as play is repeated in the finite horizon environment, trade converges to zero.' 
Harrison and McCabe (1992) report results similar to those above in a stahl-Rubinstein extensive form two-person ultimatum game. Over time 'fair' cooperative outcomes give way as the choices approach the Nash equilibrium outcome.

VI. Conclusions

The ever-present temptation to cheat (defect, free-ride) on the good offices of others in social exchange suggests that it should be difficult for cooperation and its attendant social benefits to emerge naturally in small group interaction.

Altruistic cooperation that is permissive toward cheating is therefore unstable and will be selected against in unfettered interaction. In contrast, strategies that have the general properties of $t$ it-for-tat, in the sense that cheating is punished and cooperation is reciprocated, have fitness value and are likely to persist. If this is true then the $2-3$ million year history of tool-using hominids living in small groups is likely to have produced mental modules which allow reward/punishment strategies to be learned unconsciously by individuals who maturate in any, but some, particular culture. These strategies would be learned without formal training just as children learn early in life to speak grammatically in any language if they grow up with others who speak the language, and are incapable of learning syntax if they don't. Studies of language learning 
suggest that this is because the human brain contains blue prints for language learning as part of its normal social maturation.

The large number of experimental game results summarized in this paper suggest that people invoke reward/punishment strategies in a wide variety of small group interactive contexts. These strategies are generally inconsistent with, but more profitable than, the noncooperative strategies predicted by game theory. There is, however, consistency with the game theoretic folk theorem which asserts that repetition favors cooperation, although we observe a substantial use of negative and positive reciprocity strategies, and substantial achievement of cooperative outcomes, even in single play games.

Non-cooperative outcomes are favored, however, where it is very costly to coordinate a Cooperative outcome, and in larger groups. In large groups interacting through markets using property rights and a medium of exchange, and with dispersed private information, non cooperative interaction supports the achievement of socially desirable outcomes. Experimental studies have long supported this fundamental theorem of markets. This theorem does not generally fail, however, in small group interaction because people modify their strict self-interested behavior, using reward/punishment strategies that enable some approximation of surplus maximizing outcomes. Seen in the light of evolutionary psychology, such behavior is not a puzzle, but a natural product of our mental evolution and social adaptation. 


\section{Footnotes}

We are grateful to the National Science Foundation for research support under NSF \#SBR-9210052 to the University of Arizona.

Research by neuroscientists on the amygdala, an almond sized structure deep in the temporal lobe of the brain, has shown that it is directly involved in the perception of social signals. That the amygdala participates in the social cognition and behavior of animals has been known for many years, but recent studies have shown that these findings extend to humans (Allman and Brothers, 1994; Adolphs, et al, 1994). Thus, subjects with damaged amygdalas are unable to recognize/distinguish expressions such as fear, surprise and anger on faces in photographs of people. In one study, the subject had great difficulty determining whether individuals were looking at her or away from her. The amygdala operates preconsciously: "the evidence ... clearly indicates that the amygdala is involved in the evaluation of complex stimuli long before they are completely analyzed cognitively, and probably long before they enter awareness." (Halgren, 1992, p. 194).

Pinker (1994, p. 227) for example provides the following exchange: Woman: "I'm leaving you." Man: "Who is he?" Without the ability to infer mental states from words or actions, this exchange constitutes random unrelated statements. 
other experiments have examined violations of social contracts when they do not involve cheating (Gigerenzer and Hug, cited in CT, 1992, p." 195). Only 44\% correctly solve the no cheating version, while $83 \%$ get the cheating version correct. Cosmides and Tooby (in preparation, cited in CT, 1992, pp. 198) have examined social contract problems which distinguish violations due-to cheating from violations due to innocent mistakes.. The cheating version is correctly solved by $68 \%$ of the subjects, but only $27 \%$ in the mistake version. Other social contract reasoning tasks asked subjects to detect altruists instead of cheaters. People are not good at detecting altruists. In fact where the rule was a social law (public good) more people detected cheaters than altruists. (СT, 1992, Pp. 193-195 and footnote 17). There is slippage, however, in the shift of mutual expectations when the stakes are increased to $\$ 100$. HMSa observe a small, statistically insignificant, reduction in the offer percentage in $\$ 100$ ultimate games when moving from the random/divide to the contest/exchange treatment but the rejection rate soars from 1 in 27 for $\$ 10$ ultimatums to 5 in 23 in $\$ 100$ ultimatums, an increase which is significant both statistically and economically. This outcome has since been replicated with 22 economics graduate students from across the United States attending an ESL Workshop at the University of Arizona, August 1995. Four of eleven offers were rejected in a $\$ 100$ ultimatum game: two of four $\$ 10$ 
offers, tro of five $\$ 30$ offers. "Sophistication" and training in graduate economics, including game theory, did not change negative reciprocity behavior in high stakes ultimaturim games.

5. In a repeated game player 2 can play down at $x_{6}$, forcing Player 1 to accept $(15,30)$ by moving right. By this action Dlayer 2 seeks to teach Player 1 to play right at $x_{i}$ Player 1, however, may choose rationally to escalate the punishment/counter punishment interaction by playing down at $x_{8}$ as a mezns of neutralizing Player 2's teaching strategy.

6. A move down at $x_{7}$ is irrational escalation by player 1 , since in zepeated play, Player 2 is likely to conclude that right at $x_{2}$ is the best course of action against player 1 .

7. Game theory does not, of course, literally predict SP unless the players have compete information on each other's 'types' as fully rational, calculating players. But if players do have such information then the predictions of game theory are trivialy true without the necessity of observation -knowing types is equivalent to knowing behavior.

8. The pattern of play across the 20 periods of RANDOM 1 (not shown) is significant: from the first to the last 5 trials there is an increase in the frequency of punishment by Player 25 at node $x_{5}$ from $42 \%$ to $81 \%$. This illuminates Cosmides and Tooby's (1992) design characteristic number (11) above. What is "appropriate" punishment? Most subjects accept defection early in repeated play. This is 
not unreasonable, since defection might simply be a failure of Player 1 to understand Player 2's forward message. In later plays, however, subject player 2s are much less willing to tolerate defection. These strategies work, in the sense that cooperation increases across the 20 trials.

9. Similarly, Camerer and Weigelt (1988) report very slow convergence in a sequential equilibrium reputation model. 


\section{References}

Adolphs, R., D. Tanel, H. Damasio and A. Damasio, "Impaired Recognition of Emotion in Facial Expressions Following Bilateral Damage to the Human Amygdala," Nature, 372:669672,15 December 1994.

Allman,--John-and Leslie-Brothers, "Faces, Fear and the Amygdala. Nature, 372:613-614, 15 December 1994 .

Axelrod, R., The Evolution of Cooperation. New York: Basic Books, 1984 .

Axelrod, R. and W. D. Hamilton, "The Evolution of Cooperation," Science. 211:1390-1396, 1981, Baron-Cohen, Simon, Mindblindness An Essay on Autism and Theory of Mind. Cambridge, MA: MIT Press, 1995.

Berg, J., J. Dickhaut and K. McCabe, "Trust, Reciprocity and Social History," University of Minnesota, Department of Accounting, Mimeo, October 1994.

Brewer, Marilyn and William Crano, Social Psychology, St. Paul, MN: West Publishing Co., 1994.

Camerer, Colin and Keith Weigelt, "Experimental Tests of a Sequential Equilibrium Reputation Model," Econometrica, 56:1-36, January 1988 .

Cosmides, Leda, "The Logic of Social Exchange: Has Natural Selection Shaped How Humans Reason? Studies With the Wason Selection Task," Cognition, 31:187-276, 1985. 
Cosmides, Leda and John Tooby, "From Evolution to Behavior:

Evolutionary Psychology as the Missing Link, in $J$. Dupre (ed.), The Latest and The Best: Essavs on Evolution and Optimality, Cambridge, MA: The MIT Press, 1987.

"Evolutionary Psychology and the Generation of Culture, Part II," Ethology and Sociobiology, 10:51-97, 1989. , "Cognitive Adaptations for Social

Exchange," in The Adapted Mind, edited by J. Barkow, L. Cosmides and J. Tooby, New York: Oxford University Press, 1992.

Dasgupta, P., Peter Hammond, and Eric Maskin, "The Implementation of Social Choice Rules. Some General Results on Incentive Compatibility," Review of Economic Studies, $66: 185-216$, 1979.

Davis, Douglas D. and Charles A. Holt, Experimental Economics, Princeton, NJ: Princeton University Press, 1993.

Fehr, E., G. Kirchsteiger and A. Riedl, "Does Fairness Prevent Market Clearing: An Experimental Investigation," Quarterly Journal of Economics, 108:437-459, May 1993.

Forsythe, R., J. Horowitz, N. Savin and M. Sefton, "Replicability, Fairness and Pay in Experiments with Simple Bargaining Games," Games and Economic Behavior, 1994.

Friedman, J., "A on-Cooperative Equilibrium for Supergames," Review of Economic Studies. 38:1-12, 1971. 
Fudenberg, D. and E. Maskin, "The Folk theorem With Discounting and with Incomplete Information," Econometrica, 55:533,554, 1986.

Fudenberg, Drew and Jean Tirole, Game Theory, Cambridge, MA: The MIT Press, 1993.

Green, J. and Jean-Jacques Laffont, Incentives in Public Decision Making, Amsterdam: North-Holland, . 1979.

Groves, Theodore, "Incentives in Teams," Econometrica, 41:617$631, \quad 1973$.

Halgren, Eric, "Emotional Neurophysiology of the Amygdala Within the Context of Human Cognition," in J. Aggleton, editor, The Amygdala. New York: Wiley-Liss, 1992.

Harrison, Glenn W. and Kevin A. McCabe, "Testing Noncooperative Bargaining Theory in Experiments," in R. M. Isaac, editor, Research in Experimental Economics, Vol. 5. Greenwich, Conn: JAI Press, 1992,. 137-169.

Karsanyi, J., "Games with Incomplete Information Played by Bayesian Players," Management Science. 14:159-182, 320-334, $486-502, \quad 1967-68$.

Hoffman, E., K. McCabe, K. Shachat, and V. Smith, "Preferences, Property Rights and Anonymity in Bargaining Games," Games and Economic Behavior, 1994 .

Hoffman, Elizabeth, Kevin McCabe and Vernon Smith (a), "On Expectations and the Monetary Stakes in Ultimatum Games," to appear in International Journal of Game Theory, 1995. 
(b) ," "Social Distance and other Regarding

Behavior in Dictator-Games," Economic Science Laboratory,

University of Arizona, June 1995.

Homans, G. C., The Nature of Social Sciences, New York:

Harcourt, Brace and World, 1967.

Isaac, G.L., "The Food-sharing Behavior of Protohuman Hominoids, Scientific American, 238:90-108,- 1978 .

Isaac, R. Mark, Kenneth F.McCue, and Charles R. Plott, "Public Goods Provision in an Experimental Environment," Journal of Public Economics, 26:51-74, 1985.

Isaac, R. Mark, David Schmitz, and James M. Walker, "The Assurance Problem in a Laboratory Market," Public Choice, $62: 217-236,1989$.

Isaac, R. Mark and James M. Walker, "Group Size Effects in Public Goods Provision: The Voluntary Contributions Mechanism," Quarterly Journal of Economics, 103:179-200, 1988a. "Communication and Free-Riding Behavior:

The Voluntary Contributions Mechanism," Economic Inquiry, $26: 585-608,1988 \mathrm{~b}$.

"Costly Communication: An Experiment in a Nested Public Goods Problem," in T. Palfrey (ed.), Contemporary Laboratory Research in Political Economy, Ann Arbor: University of Michigan Press, 1991.

Isaac, R. Mark, James M. Walker, and Susan H. Thomas, "Divergent Evidence on Free Riding: An Experimental Examination of Possible Explanations," Public Choice, 43:113-149, 1984. 
Isaac, R. Mark, James M. Walker, and Arlington Williams, "Group Size and the Voluntary Provision of Public Goods:

Experimental Evidence Utilizing Large Groups," Indiana.. University Working Paper, 1991.

Kahneman, Daniel, Jack Knetsch and Richard Thaler, "Fairness and the Assumptions of Economics," Journal of Business 59, Part 2,. October 1986, S285-S300.

Kaplin, H. and K. Hill, "Food Sharing Among Ache Foragers: Test of Explanatory Hypotheses," Current Anthropology, 26:223246, March 1985.

Kreps, D., P. Milgrom, J. Roberts and R. Wilson, "Rational Cooperation in the Finitely Repeated Prisoners' Dilemma," Journal of Economic Theory, 27:245-252, 1982 .

Laffont, 1994.

Lee, R.B. and I. De Vore (eds.) Man the Hunter, Chicago: Aldine, 1968.

Macfarlane, Alan, The Origins of English Individualism. New York: Cambridge University Press, 1978.

Marr. D., Vision. San Francisco, CA: Freeman, 1982.

McCabe, Kevin, "Fiat Money as a Store of Value' in an Experimental Market," Journal of Economic Behavior and Organization, 12:215-231, October 1989 .

McCabe, Kevin, Stephen Rassenti and Vernon Smith, "Forward and Backward Rationality in Achieving Cooperation," Economic Science Laboratory, University of Arizona, August 1994. 
Osborne," Martin J . and Ariel Rubinstein, A Course in Game Theory, Cambridge, MA: The MIT Press, 1994.

Ostrom, Elinor, Roy Gardner, and James Walker, Rules, Games, and Common Pool Resources. Ann Arbor, MI: The University of Michigan Press, 1994.

Pinker, Steven, The Language Instinct: How the Mind Creates Lanugage. London: Viking, 1994

Porter, Dave and Vernon Smith (a), "Stock Markets in the Laboratory," to appear Applied Mathematical Finance, 1995. Rapoport, A., "Prisoner's Dilemma," in The New Palgrave, Volume 3, pp. 97 3-97 6, J. Eatwell, M. Milgate and P. Newman eds., London: Macmillan, 1987.

Roth, A., V. Prasniker, M. Okuno-Fujimara and S. Zamir, "Bargaining and Market Behavior in Jerusalem, Ljubligana, Pittsburgh and Tokyo: An Experimental Study," American Economic Review, 81:1068-1095, 1991.

Schotter, Andrew, Keith Wiegelt and Charles Wilson, "A Laboratory Investigation of Multiperson Rationality and Presentation Effects," Games and Economic Behavior, 6:445-468, 1994 .

Selten, R., "Reexamination of the Perfectness Concept for

Equilibrium Points in Extensive Games," International Journal of Game Theory, 4:25-55, 1975.

Selten, R. and R. Stoecker, "End Behavior in Sequences of Finite Prisoner's Dilemma Supergames," journal of Economic Behavior and Organization. 7:47-70, 1986. 
Smith, Vernon L., Gerry L. Suchanek, and Arlington W. Williams, "Bubbles, Crashes and endogenous Expectations in Experimental Spot Asset Markets," Econometrica, 56:11191151, September 1988 .

Steven, The Language Instinct. New York: William Morrow and Co., Inc., 1994 .

Tooby John and De Vore, "The Reconstruction of Hominoid 3ehavioral Evolution Through Strategic Modelling," in W. G. Kinzey (ed.), Primate Models of Human Behavior, New York; SUNY Press, 1987 .

Trivers R.L., "The Evolution of Reciprocal Altruism," Quarterly Review of Biology. 46:35-57, 1971 .

Wason, P., "Reasoning," in B. M. Foss, editor, New Horizons in Psychology, Harmondsworth: Penguin, 1966. 
Figure $3(x)$

Ultimatum; Random Entitlement, FHSS Instructions, Divide $\$ 10, \mathrm{~N}=\mathbf{2 4}$

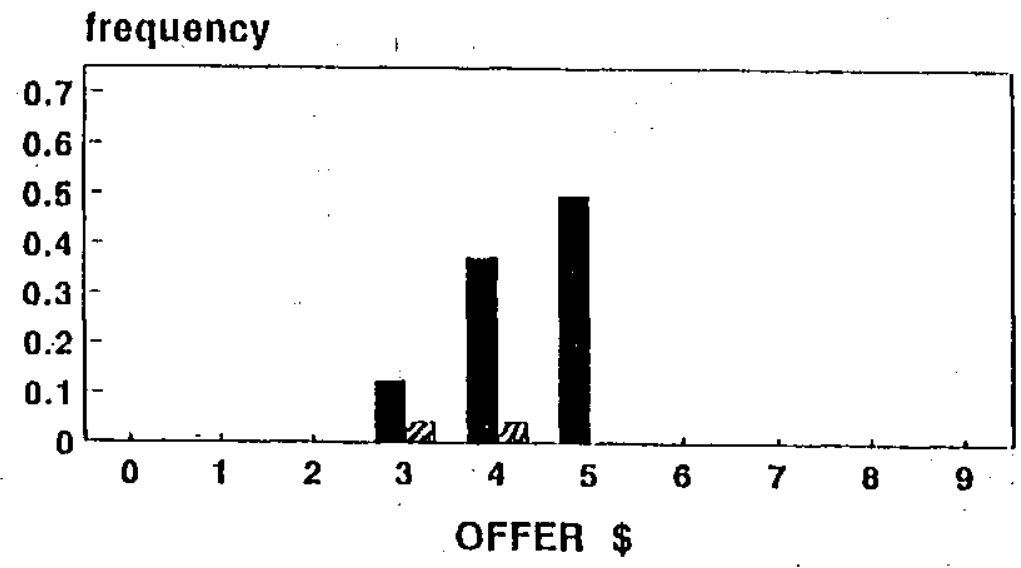

$11 \%$ OFFER $0 \%$ REJECTION

Figure 3(c)

Ultimatum; Random Entitlement,

Exchange, $\mathrm{N}=24$

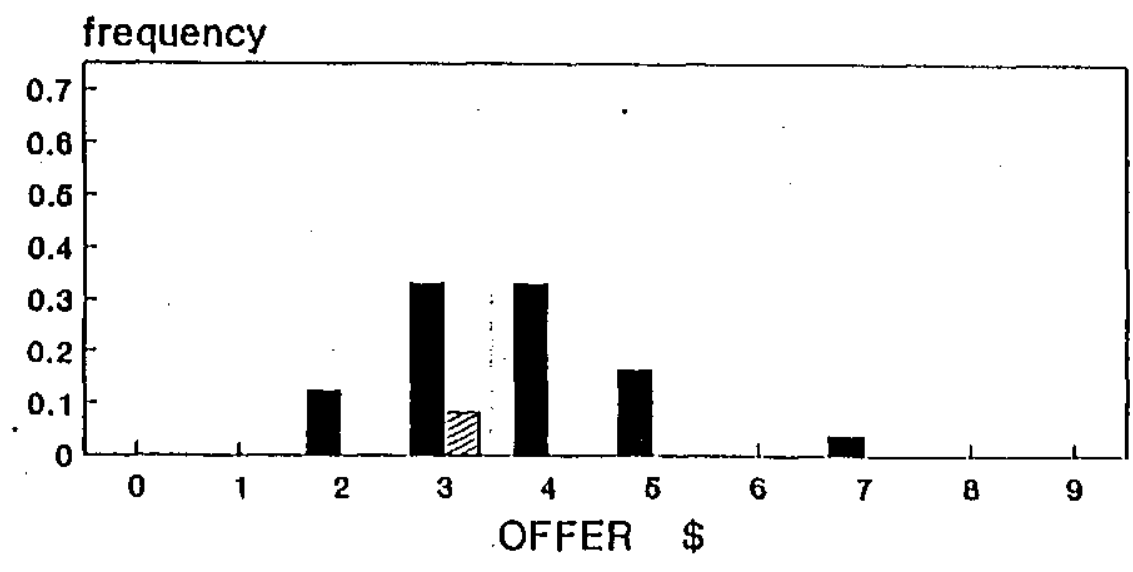

OFFER \% REJECTIONS
Figure ${ }^{3(b)}$

Ultimatum; Contest Entitlement, FHSS Instructions, Divide $\$ 10, N=24$

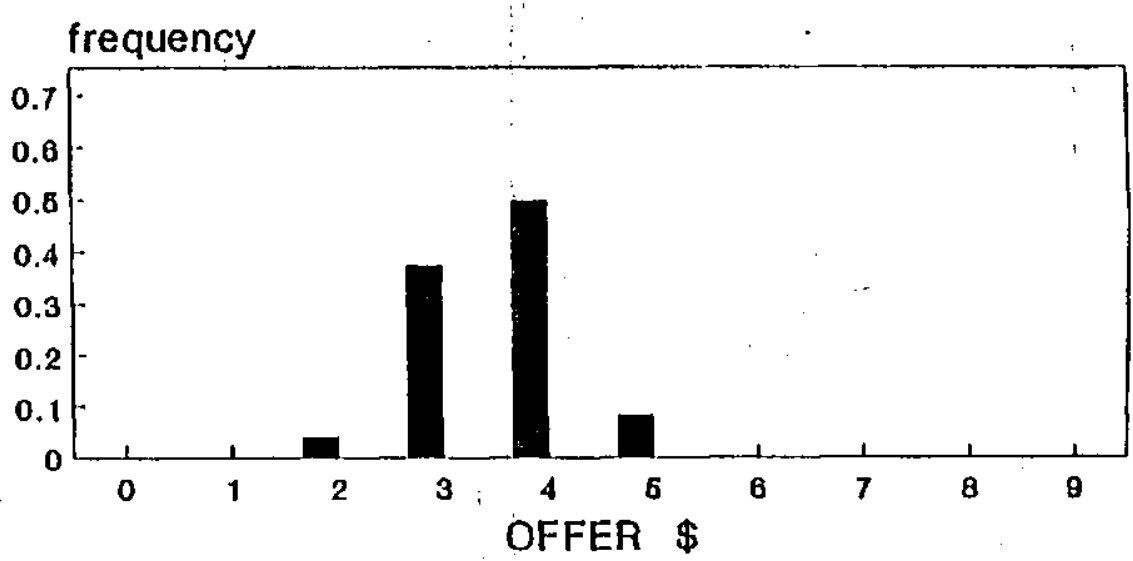

\% OFFER O REJECTION

Figure ${ }^{3}(\mathrm{~d})$

Ultimatum; Contest Entitlement,

Exchange, $N=24$

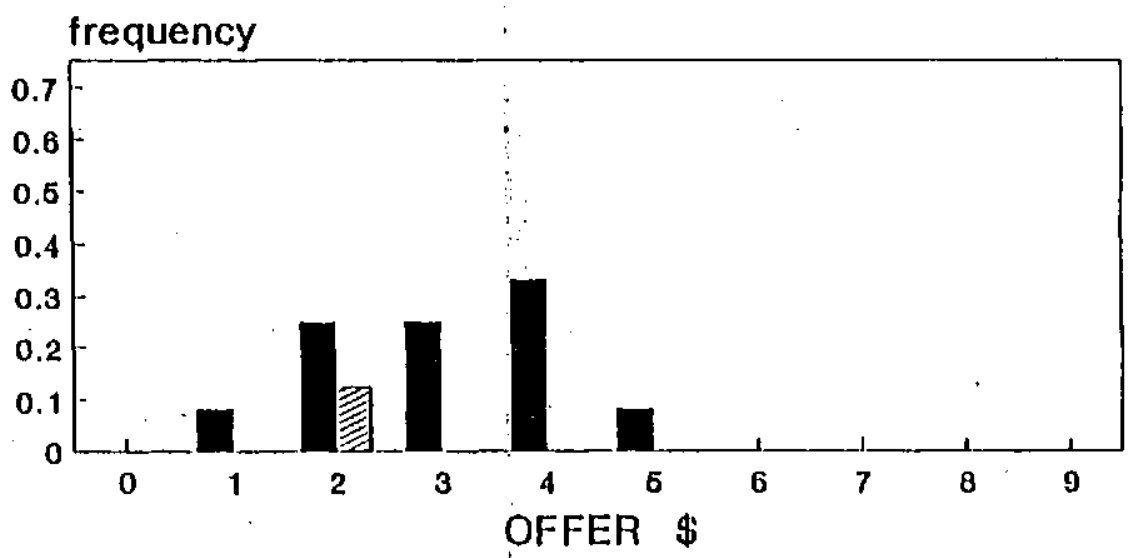

\% OFFERS \% REJECTIONS 
Contest/Exchange Results

From HMSS, $\mathrm{N}=24$

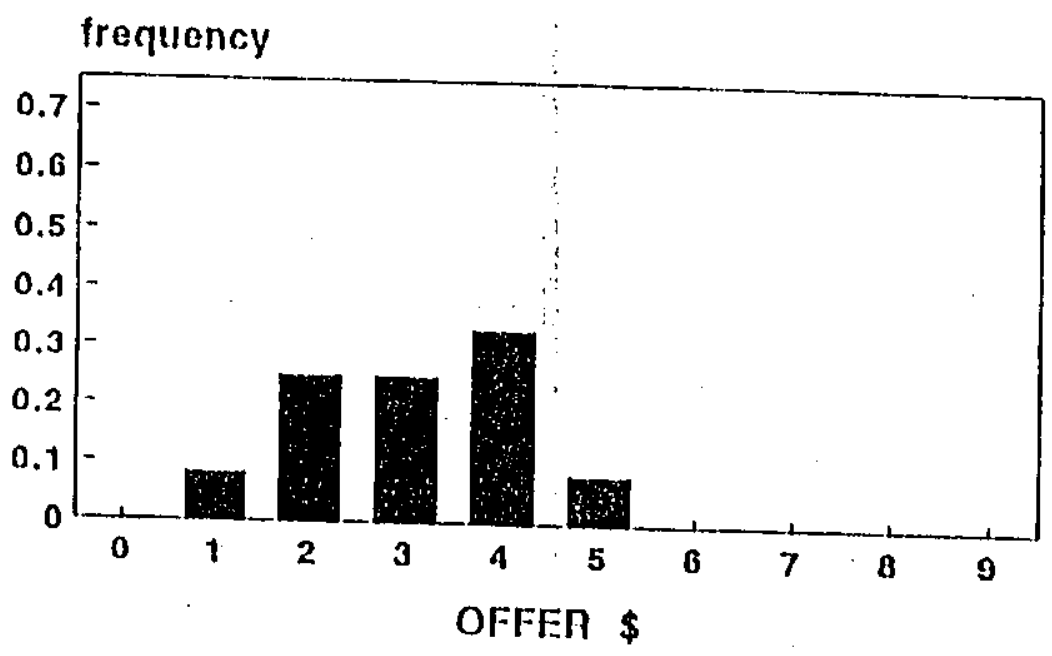

Contest/Exchange With Added Instructions Attached; $\mathrm{N}=24$

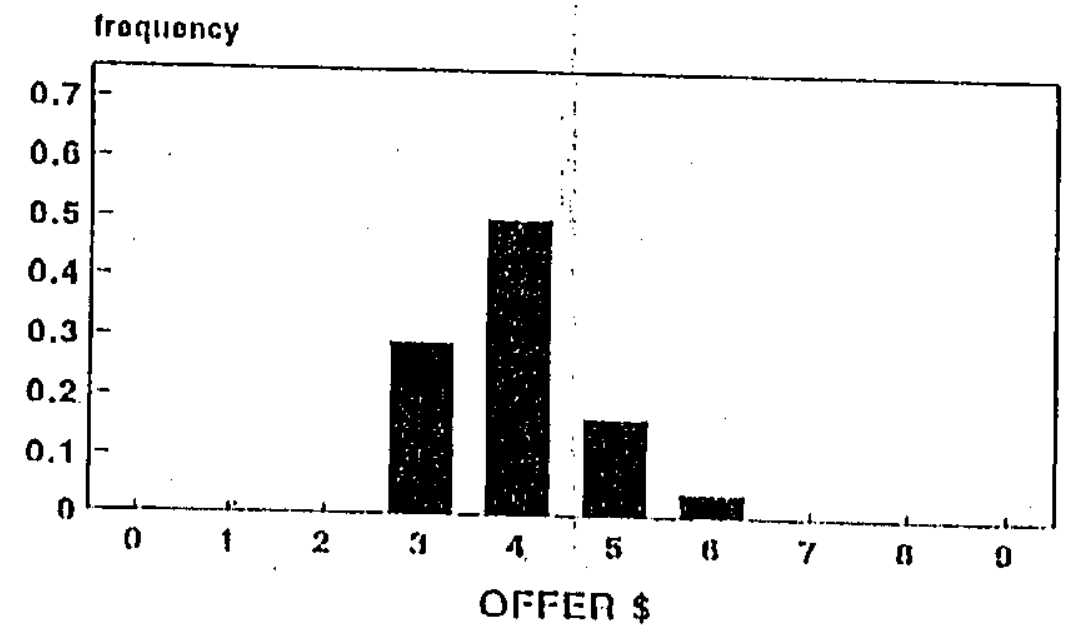

\section{Randlom/Exchange Results}

From HMSS, $\mathrm{N}=24$

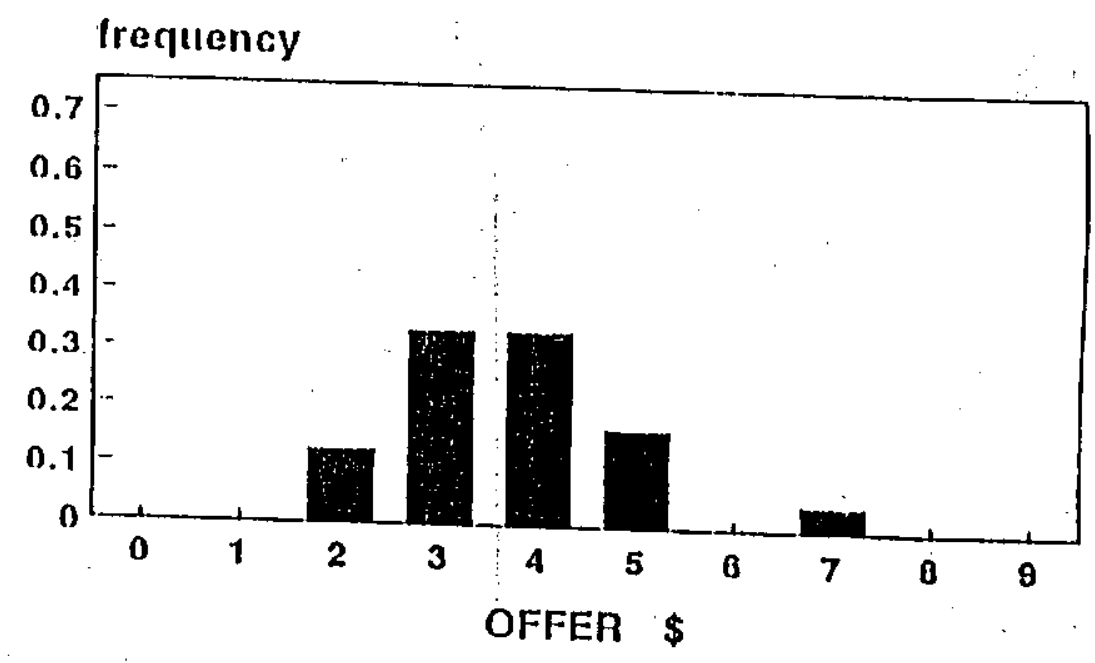

Random/Exchange With Added Instructions Attached, $\mathrm{N}=24$

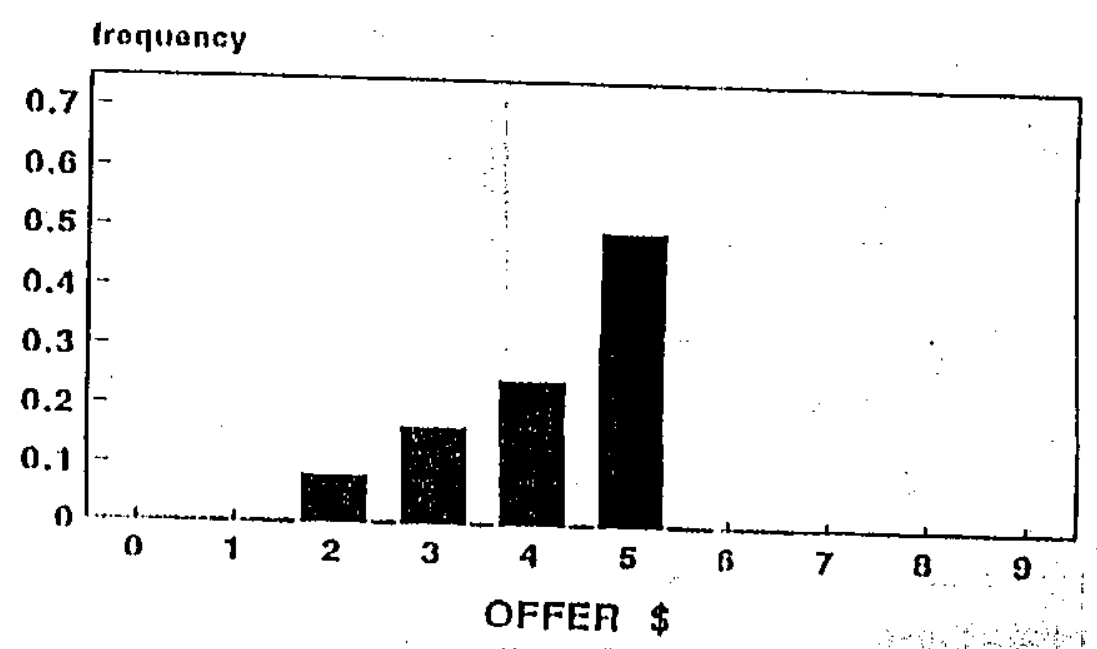




\section{Figure 5}

\section{Extensive Form Eame Tree Games $1 *$ and 2}

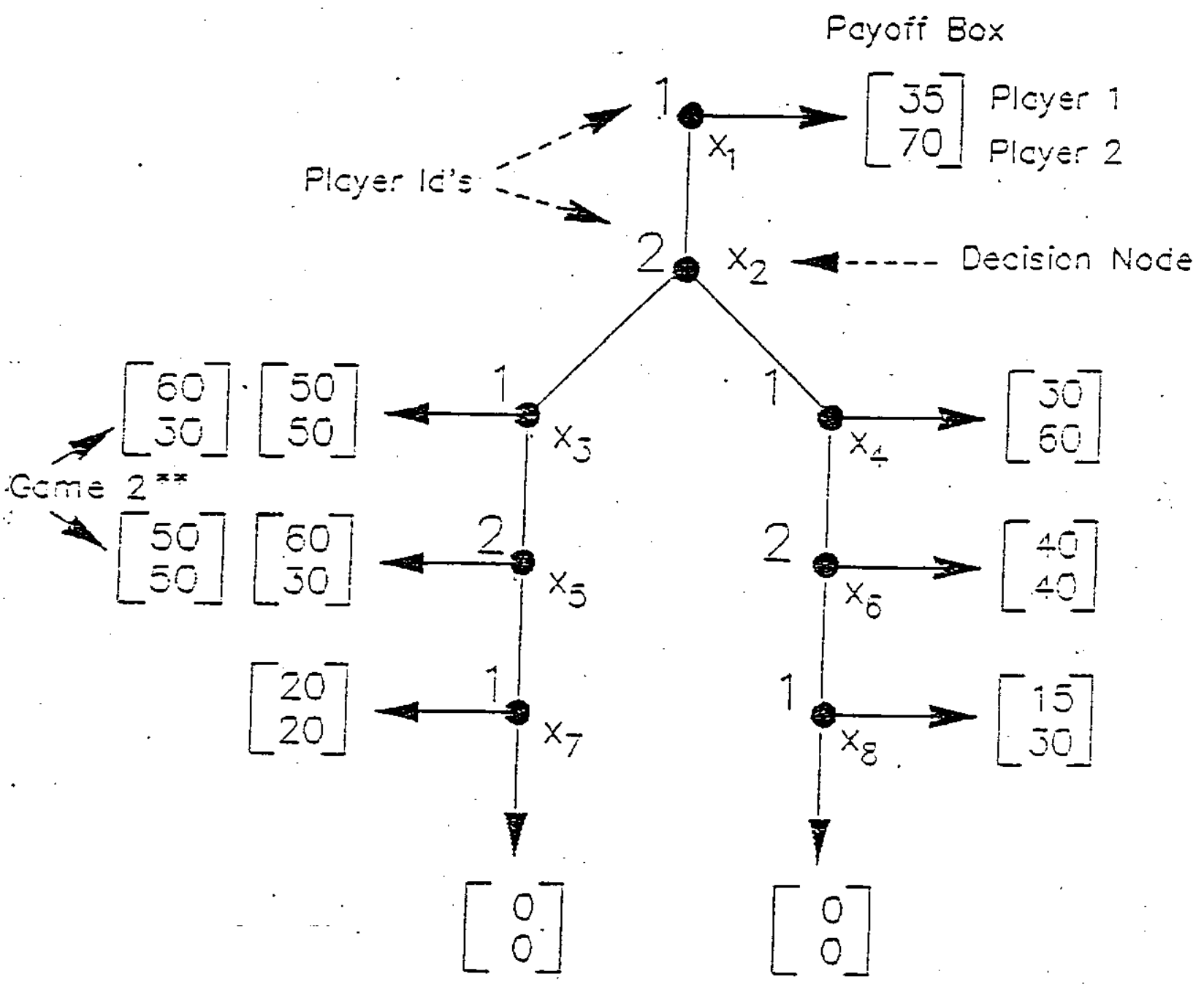

* Gome 1 poyofís cre closest to gome tree.

* Gome 2 difiers irom Gome 1 by thase two poyoî́ boxes. 
Table 1

Epps-Singleton Test

(Ievel of significance)

\begin{tabular}{|c|c|c|c|}
\hline & $\begin{array}{l}\text { HMMS Contest } \\
\text { Exchange }\end{array}$ & $\begin{array}{l}\text { Random } \\
\text { Exchange Added } \\
\text { Instructions }\end{array}$ & $\begin{array}{l}\text { Contest } \\
\text { Exchange Added } \\
\text { Instructions }\end{array}$ \\
\hline $\begin{array}{l}\text { FMSS Rancom } \\
\text { Exchange }\end{array}$ & $\begin{array}{c}3.88 \\
(0.42) \\
\end{array}$ & $\begin{array}{c}6.28 \\
(0.18) \\
\end{array}$ & $\begin{array}{c}4.1 \\
(0.4) \\
\end{array}$ \\
\hline $\begin{array}{l}\text { FMSS Contest } \\
\text { Exchange }\end{array}$ & $\therefore$ & $\begin{array}{l}12.4 \\
(0.01)\end{array}$ & $\begin{array}{l}10.0 \\
(0.04)\end{array}$ \\
\hline $\begin{array}{l}\text { Random Exciange } \\
\text { Addec } \\
\text { Instructions }\end{array}$ & & & $\begin{array}{c}9.75 \\
(0.045)\end{array}$ \\
\hline
\end{tabular}


Table 2

Wilcoxon Rank-Sum Test

(level of significance)

\begin{tabular}{|c|c|c|c|}
\hline & $\begin{array}{l}\text { FMMS Contest } \\
\text { Exchange }\end{array}$ & $\begin{array}{l}\text { Random } \\
\text { Exchange Added } \\
\text { Instructions }\end{array}$ & $\begin{array}{l}\text { Contest } \\
\text { Exchange Added } \\
\text { Instructions }\end{array}$ \\
\hline $\begin{array}{l}\text { HMSS Random } \\
\text { Exchange }\end{array}$ & $\begin{array}{l}-1.66 \\
(0.10) \\
\end{array}$ & $\begin{array}{c}1.90 \\
(0.06)\end{array}$ & $\begin{array}{c}1.07 \\
(0.29) \\
\end{array}$ \\
\hline $\begin{array}{l}\text { HMSS Contest } \\
\text { Exchange }\end{array}$ & . & $\begin{array}{r}3.34 \\
(0.00) \\
\end{array}$ & $\begin{array}{l}2.7 \\
(0.01) \\
\end{array}$ \\
\hline $\begin{array}{l}\text { Random Exchange } \\
\text { Added } \\
\text { Instructions }\end{array}$ & $\cdots$ & & $\begin{array}{l}-1.26 \\
(0.21)\end{array}$ \\
\hline
\end{tabular}


Table 3

Experimental Design

Treatments and Number of Pairs

\begin{tabular}{|c|c|c|c|}
\hline Designation & mstituent Game & Matching Protocol & airs ${ }^{c}$ \\
\hline SINGLE 1 & 1 & SINGLE PLAY & 26 \\
\hline SINGLE 2 & 1 & SINGLE PLAY & 17 \\
\hline REPEAT SINGLE 1 & 1 & REPEAT SINGLE PLAY' & 26 \\
\hline SINGLE 1 EXPERIENCED & 1 & SINGLE PLAY & 17 \\
\hline SINGLE 1 CONTINGENT & 1 & SINGLE PLAY, ALL NODES & 24 \\
\hline 'RANDOM 1 & 1 & RANDOM PAIRS, ROLES & 24 \\
\hline RANDOM 2 & 2 & RANDOM PAIRS, ROLES & 24 \\
\hline SAME 1 & 1 & SAME PAIRS & 22 \\
\hline SAME 2 & 2 & SAME PAIRS & 23 \\
\hline
\end{tabular}

a. Payoff information is complete -- both players know both payoffs in all treatments.

b. Each subject plays each counterpart once will type alternating between player 1 and 2.

c. Sessions consist of 12 subjects, 6 pairs matched repeatedly except in SAME 1 and 2, SINGLE 1, and REPEAT SINGLE 1.

d. Contingent play means that each player makes a response at each of his/lier decision nodes. Then the computer executes the play once. 
Table 4

\section{Summary Data; All Treatments}

\begin{tabular}{|c|c|c|c|c|c|c|c|c|c|c|c|c|c|c|}
\hline & \multicolumn{14}{|c|}{ Conditional Oulcome Probabilities by Trealment; for all Trials } \\
\hline Treilment & $\begin{array}{l}35 \\
70 \\
\end{array}$ & Len & $\begin{array}{r}50 \\
50 \\
\end{array}$ & $\begin{array}{r}60 \\
30 \\
\end{array}$ & $\begin{array}{l}20 \\
20 \\
\end{array}$ & $\begin{array}{l}0 \\
0 \\
\end{array}$ & Right & $\begin{array}{l}30 \\
60 \\
\end{array}$ & $\begin{array}{r}40 \\
40 \\
\end{array}$ & $\begin{array}{l}15 \\
30 \\
\end{array}$ & $\begin{array}{l}0 \\
0\end{array}$ & $\begin{array}{l}E\left(\pi_{2}\right) \\
\text { Leff })^{c}\end{array}$ & $\begin{array}{l}E\left(\pi_{1}\right) \\
\text { Dowvin) }\end{array}$ & $\underset{*}{\text { Efficiency }}$ \\
\hline Singlc I & ( & $\begin{array}{l}13 / 26 \\
=.50 \\
\end{array}$ & $\begin{array}{l}10 / 13 \\
=.769\end{array}$ & $\begin{array}{l}2 / 3 \\
=.667 \\
\end{array}$ & $\begin{array}{l}1 / 1 \\
=1 \\
\end{array}$ & 0 & $\begin{array}{l}13 / 26 \\
=.50 \\
\end{array}$ & $\begin{array}{l}0 / 13 \\
=0\end{array}$ & $\begin{array}{l}12 / 13 \\
=.92\end{array}$ & $\begin{array}{l}1 / 1 \\
=1\end{array}$ & 0 & 44.6 & 46.7 & 85.5 \\
\hline Single $2^{\prime}$ & 0 & $\begin{array}{l}12 / 26 \\
=.462 \\
\end{array}$ & $\begin{array}{l}6 / 6 \\
=1 \\
\end{array}$ & $\begin{array}{l}6 / 12 \\
=.5 \\
\end{array}$ & II & 0 & $\begin{array}{l}11 / 26 \\
=.538 \\
\end{array}$ & $\begin{array}{l}0 / 14 \\
=0\end{array}$ & $\begin{array}{l}14 / 14 \\
=1\end{array}$ & 0 & 0 & 40.0 & -.. & 86.9 \\
\hline $\begin{array}{l}\text { Repent } \\
\text { Single I }\end{array}$ & $\begin{array}{r}8 / 360 \\
=.022 \\
\end{array}$ & $\begin{array}{l}204 / 352 \\
\simeq .580 \\
\end{array}$ & $\begin{array}{l}133 / 204 \\
=.652 \\
\end{array}$ & $\begin{array}{l}33 / 71 \\
=.549 \\
\end{array}$ & $\begin{array}{l}36 / 36 \\
=1 \\
\end{array}$ & $\mathbf{0}$ & $\begin{array}{l}148 / 352 \\
=.420 \\
\end{array}$ & $\begin{array}{l}9 / 148 \\
=.061 \\
\end{array}$ & $\begin{array}{l}138 / 139 \\
=.993\end{array}$ & $\begin{array}{l}0 / 1 \\
=0 \\
\end{array}$ & $\begin{array}{l}1 / 1 \\
=1\end{array}$ & 41.5 & 42.0 & 85.1 \\
\hline $\begin{array}{l}\text { Single I } \\
\text { expcri- } \\
\text { enced }\end{array}$ & 0 & $\begin{array}{l}13 / 17 \\
=.765\end{array}$ & $\begin{array}{l}8 / 13 \\
=.613\end{array}$ & $\begin{array}{l}3 / 5 \\
=.60\end{array}$ & $\begin{array}{l}2 / 2 \\
=1\end{array}$ & $\mathbf{0}$ & $\begin{array}{l}4 / 17 \\
=.235\end{array}$ & $\begin{array}{l}0 / 4 \\
=0\end{array}$ & $\begin{array}{l}4 / 4 \\
=1\end{array}$ & 0 & 0 & 40.7 & 44.0 & 86.4 \\
\hline $\begin{array}{l}\text { Single } 1 \\
\text { contingent }\end{array}$ & $\begin{array}{l}1 / 24 \\
=.042 \\
\end{array}$ & $\begin{array}{l}9 / 23 \\
=: 391 \\
\end{array}$ & $\begin{array}{l}8 / 9 \\
=.889 \\
\end{array}$ & $\begin{array}{l}1 / 9 \\
=.111\end{array}$ & 0 & 0 & $\begin{array}{r}14 / 23 \\
=.609 \\
\end{array}$ & $\begin{array}{l}3 / 14 \\
=.214 \\
\end{array}$ & $\begin{array}{l}11 / 11 \\
=1\end{array}$ & $\mathbf{0}$ & 0 & 47.8 & 6.7 & 88.7 \\
\hline Random I & $\begin{array}{r}9 / 480 \\
=.019 \\
\end{array}$ & $\begin{array}{l}317 / 471 \\
=.673\end{array}$ & $\begin{array}{l}215 / 317 \\
=.678 \\
\end{array}$ & $\begin{array}{l}33 / 102 \\
=.324 \\
\end{array}$ & $\begin{array}{l}58 / 69 \\
=.811 \\
\end{array}$ & $\begin{array}{l}11 / 69 \\
=.159 \\
\end{array}$ & $\begin{array}{l}154 / 471 \\
=.327 \\
\end{array}$ & $\begin{array}{l}1 / 154 \\
=.006 \\
\end{array}$ & $\begin{array}{l}149 / 153 \\
=.974\end{array}$ & $\begin{array}{l}3 / 4 \\
=.75 \\
\end{array}$ & $\begin{array}{l}1 / 4 \\
=.25\end{array}$ & 40.7 & 30.8 & 82.6 \\
\hline Random 2" & $\begin{array}{r}27 / 480 \\
=.056 \\
\end{array}$ & $\begin{array}{l}16(0 / 453 \\
=.353\end{array}$ & $\begin{array}{l}90 / 90 \\
=1\end{array}$ & $\begin{array}{r}70 / 160 \\
=.438 \\
\end{array}$ & 0 & 0 & $\begin{array}{l}293 / 453 \\
=.647 \\
\end{array}$ & $\begin{array}{l}7 / 293 \\
=.024 \\
\end{array}$ & $\begin{array}{l}278 / 286 \\
=.972 \\
\end{array}$ & $\begin{array}{l}4 / 8 \\
=.50 \\
\end{array}$ & $\begin{array}{l}4 / 8 \\
=.50 \\
\end{array}$ & 41.2 & $\therefore$ & 84.7 \\
\hline Sasurc 1 & $\begin{array}{l}7 / 440 \\
=.016 \\
\end{array}$ & $\begin{array}{l}353 / 433 \\
=.815 \\
\end{array}$ & $\begin{array}{l}312 / .153 \\
=.884 \\
\end{array}$ & $\begin{array}{l}11 / 41 \\
=.341 \\
\end{array}$ & $\begin{array}{r}22 / 27 \\
=.815\end{array}$ & $\begin{array}{l}5 / 27 \\
=.185 \\
\end{array}$ & $\begin{array}{l}80 / 4.33 \\
=185 \\
\end{array}$ & $\begin{array}{l}3 / 80 \\
=.038 \\
\end{array}$ & $\begin{array}{l}68 / 77 \\
=.883 \\
\end{array}$ & $\begin{array}{l}4 / 9 \\
=.444 \\
\end{array}$ & $\begin{array}{l}5 / 9 \\
=.556\end{array}$ & 46.6 & 31.2 & 90.6 \\
\hline Sante 2 & $\begin{array}{l}37 / 460 \\
=.080 \\
\end{array}$ & $\begin{array}{l}261 / 423 \\
=.617 \\
\end{array}$ & $\begin{array}{l}220 / 220 \\
=1\end{array}$ & $\begin{array}{l}41 / 261 \\
=.157\end{array}$ & 0 & 0 & $\begin{array}{l}162 / 423 \\
=383\end{array}$ & $\begin{array}{l}27 / 162 \\
=.167\end{array}$ & $\begin{aligned} & 114 / 135 \\
&= 844 \\
&\end{aligned}$ & $\begin{array}{l}21 / 21 \\
=1\end{array}$ & 0 & $46 . .9$ & $\cdots$ & 90.3 \\
\hline
\end{tabular}

a. Note that in Game 2 the play order of the outcomes $(60,30)$ and $(50,50)$ are reversed relative to gane 1 .

Expected payef 24 pairs from Random I returned for a single play of game I for a 20-fold increase in the game I payoffs.

Expecied payof to player 2 from playing len at $x_{2}$, given the relative frequencies of subsequent play by player 1 and 2.

d. Expecied payoff to player I from defecling at node $x_{3}$.

c. Efliciency is the percent of thic cooperative $(50,50)$ total payofr that is realized by all pairs. 